\title{
Holographic estimation of multiplicity and membranes collision in modified spaces $\mathrm{AdS}_{5}$
}

\author{
I.Ya. Aref'eva ${ }^{1 *}$, E.O. Pozdeeva ${ }^{2 \dagger}$, T.O. Pozdeeva ${ }^{3 \ddagger}$ \\ ${ }^{1}$ Steklov Mathematical Institute of Russian Academy of Science, Moscow, Russia \\ 2 Skobeltsyn Institute of Nuclear Physics, Lomonosov Moscow State University, Moscow, Russia \\ ${ }^{3}$ Moscow Aviation Institute (National Research University), Moscow, Russia
}

\begin{abstract}
The quark-gluon plasma formed as a result of heavy-ion collisions is currently investigated actively both theoretically and experimentally. According to the holographic approach, forming a quark-gluon plasma in the four-dimensional world is associated with creating black holes in a five-dimensional anti-de Sitter space. The multiplicity of particles produced in heavy-ion collisions is then determined by the entropy of the five-dimensional black hole, which is estimated by the area of the trapped surface. To fit the experimental data for multiplicity Kiritsis and Taliotis have proposed to consider black holes formation in modified $\mathrm{AdS}_{5}$ spaces with different $b$-factors. In this paper we consider the formation of black holes under collision of membranes in modified $\mathrm{AdS}_{5}$ spaces with $b$-factors. Following the previous proposals we consider the power-law and exponential $b$-factors, as well as mixed types of $b$-factors. We study dynamics of the change of the trapped surface area depending on the energy for each investigated space. We find that the power-law and mixed factors fit better to the experimental date.
\end{abstract}

Keywords: anti-de Sitter space, black hole, trapped surface, heavy ion collision, particle creation multiplicity, membrane collision.

\section{Introduction}

The AdS/CFT duality is a powerful method for studying quantum systems in situations where the ordinary perturbation theory is inapplicable [1]-[3], for the recent review see [4]. The description of the quark-gluon plasma (QGP) formation in heavy-ion collisions using the idea of AdS/CFT duality has recently been actively developed, see [5] and refs there in. The QGP formation process (thermalization) is then interpreted as a black hole formation process in an auxiliary five-dimensional anti-de Sitter $\left(\mathrm{AdS}_{5}\right)$ space. The formation of black holes in the $\mathrm{AdS}_{5}$ space is considered both using the analysis of shock waves [6]-[14] and using the

\footnotetext{
*E-mail: arefeva@mi.ras.ru

${ }^{\dagger}$ E-mail: pozdeeva@www-hep.sinp.msu.ru

${ }^{\ddagger}$ E-mail: pozdeeva@inbox.ru
} 
Vaidya metric (see [15]-18] and the references therein). Such a method allows deriving the physical characteristics of the quantum four-dimensional system based on results obtained in the $\mathrm{AdS}_{5}$ space for a classical system. In particular, one can get the holographic estimation of the multiplicity of particle production in heavy-ion collisions. The multiplicity is assumed to be determined by the entropy of the black hole created in the $\mathrm{AdS}_{5}$ space. This hypothesis allows estimating the dependence of the multiplicity on the energy and comparing it with the experimental data already obtained [19].

The elementary dual models considered in [6]-[13] require modification [14] to describe the experimental data more precisely. The problem of black hole formation for modified models has been considered by Kiritsis and Taliotis in the case of point-like sources [14. However these calculations are rather complicated, and it is therefore interesting to use domain walls as a model of colliding ions. This approach was proposed in [10]. A set of problems associated with the infinite sizes of the domain walls then arises, but the regularization in which a finite wall size is introduced, as we showed in [13], allows using this method. Using domain walls significantly simplifies the problem for modified models.

In this paper we consider domain-walls (membranes) collision in a modified $\mathrm{AdS}_{5}$ space. We follow the modifications proposed in [20]-22]. The basis for the proposed modifications is the introduction of $b$-factors of the power-law, exponential, and mixed types. Our main goal here is to estimate the dependence of the trapped surface area on the energy of colliding walls for different types of $b$-factors and use this estimation to get dependence of the multiplicity on the energy of colliding ions.

The paper is organized as follows. In Sect. 2 we remind the main facts about the $b$-factor modification of $\mathrm{AdS}_{5}$. Here we consider power-law, exponential and mixed types $b$-factor. In Sect. 2.1 we pay a special attention to potentials of the scalar field responsible for the given $b$-factors. Then in Sect. 2.2 and Sect. 2.3 we present the shock waves for point-like sources as well as for domain walls in these modified $\mathrm{AdS}_{5}$ spaces. In the end of our set-up we present equations defined the trapped surface. In Sect. 3 we perform as estimations of the area of the trapped surface produce in collisions of two shock domain walls in the modified $\mathrm{AdS}_{5}$ with the power-law $b$-factor. In Sect. 7 we do the same for the exponential $b$-factor. In Sect. 5 and Sect. 6 we consider the mixed $b$-factors.

\section{Set up}

\subsection{Background}

We consider the action of five-dimensional gravity coupled to a scalar dilaton field in the presence of a negative cosmological constant

$$
S_{5}=S_{R}+S_{\Phi}
$$

here, $S_{R}$ is the Einstein-Hilbert action with the negative cosmological constant

$$
S_{R}=-\frac{1}{16 \pi G_{5}} \int \sqrt{-g}\left[R+\frac{d(d-1)}{L^{2}}\right] d x^{5},
$$

$d+1=D=5, S_{\phi}$ is the dilaton action,

$$
S_{\Phi}=-\frac{1}{16 \pi G_{5}} \int \sqrt{-g}\left[-\frac{4}{3}(\partial \Phi)^{2}+V(\Phi)\right] d x^{5} .
$$


It is assumed that the background metric has the form

$$
d s^{2}=b^{2}(z)\left(d z^{2}+d x^{i} d x^{i}-d x^{+} d x^{-}\right), \quad i=1,2,
$$

In this paper we consider several types of $b$-factors [14]. The Einstein equations reduce to two independent relations [20, 21].

$$
\begin{gathered}
\frac{3 b^{\prime \prime}}{b}+\frac{2}{3}\left(\Phi^{\prime}\right)^{2}-\frac{b^{2}}{2} V(\Phi)-\frac{6 b^{2}}{L^{2}}=0, \\
\frac{6\left(b^{\prime}\right)^{2}}{b^{2}}-\frac{2}{3}\left(\Phi^{\prime}\right)^{2}-\frac{b^{2}}{2} V(\Phi)-\frac{6 b^{2}}{L^{2}}=0,
\end{gathered}
$$

where $b=b(z), b^{\prime}=\partial_{z} b$ and the dilaton field depends only on $z, \Phi=\Phi(z)$.

The scalar field equation is

$$
\frac{1}{b^{5}} \frac{\partial}{\partial z}\left(b^{3} \frac{\partial}{\partial z}\right) \Phi+\frac{3}{8} \frac{\partial V\left(\Phi_{s}\right)}{\partial \Phi_{s}}=0 .
$$

One can see from (3) and (44) that the dilaton field and its potential are related to the $b$-factor:

$$
\begin{aligned}
& \Phi^{\prime}= \pm \frac{3}{2} \sqrt{\left(\frac{2\left(b^{\prime}\right)^{2}}{b^{2}}-\frac{b^{\prime \prime}}{b}\right)}, \\
& V(\Phi(z))=\frac{3}{b^{2}}\left(\frac{b^{\prime \prime}}{b}+\frac{2\left(b^{\prime}\right)^{2}}{b^{2}}-\frac{4 b^{2}}{L^{2}}\right) .
\end{aligned}
$$

Note also that equations (77) and (66) guarantee the validity of the field equation (5).

These equations for the given $b \neq b(z)$ provide a non-explicit form of potential $V=V(\Phi)$. The explicit form $V=V(\Phi)$ can be found by the superpotential method [22]. To use this method it is convenient to rewrite (44) and (5) in the domain wall coordinates 1 ]

$$
u=\int b(z) d z
$$

and metric has the form

$$
d s^{2}=d u^{2}+e^{2 A(u)}\left(d x^{i} d x^{i}-d x^{+} d x^{+}\right),
$$

where $A(u)=\ln (b(u))$. The potential is related with the superpotential $W$ as

$$
V(\Phi)=-\frac{4}{3}\left(\frac{d W}{d \Phi}\right)^{2}+\frac{64}{27} W^{2}-\frac{12}{L^{2}} .
$$

The superpotential is defined from equation the following

$$
W(\Phi)=-\frac{9}{4} \frac{d A}{d u}
$$

and the dilaton field is given by

$$
\Phi= \pm \frac{3}{2} \int \sqrt{-\frac{d^{2} A}{d u^{2}}} d u
$$

\footnotetext{
${ }^{1} u$ is an analogue of the cosmic coordinate in cosmological application of the superpotential method, see [23].
} 


\subsubsection{Exponential $b$-factor}

We begin by a space with $b$-factor of the form $b=\mathrm{e}^{-z / R}$. It is assumed that $R \sim \Lambda_{\mathrm{QCD}}^{-1} \sim 1 \mathrm{fm}$.

With the help of superpotentials method one gets the dependence of the potential from the field

$$
V(\Phi)=-\frac{12}{L^{2}}+\frac{9}{R^{2}} \exp \left( \pm \frac{4\left(\Phi_{s}-\Phi_{0}\right)}{3}\right), \quad \text { for } \quad b=\mathrm{e}^{-z / R}
$$

where $\Phi_{0}$ is a constant.

\subsubsection{Power-law $b$-factor}

Now we consider a space with power-law $b$-factor of the form $b(z)=\left(\frac{L}{z}\right)^{a}$. If $a=1$ we have $\mathrm{AdS}_{5}$ space.

The potential and fields can be represented explicitly thought variable $z$. Since in this case $\Phi=\Phi(z)$ is single-valued function we can find $z=z(\Phi)$ and substitute it to the expression for potential $V(z)$ to get

$$
V(\Phi)=-\frac{12}{L^{2}}+\frac{3 a(3 a+1)}{L^{2 a}} \exp \left( \pm \frac{4}{3} \sqrt{\frac{(a-1)}{a}}\left(\Phi-\Phi_{0}\right)\right) .
$$

$V(\Phi)$ is real for $a>1$.

To give a meaning to $b(z)=\left(\frac{L}{z}\right)^{a}$ with $a<1$ one can consider the phantom field $\Phi_{p}$ with the action

$$
S_{\Phi_{p}}=-\frac{1}{16 \pi G_{5}} \int \sqrt{-g}\left[\frac{4}{3}\left(\partial \Phi_{p}\right)^{2}+\tilde{V}\left(\Phi_{p}\right)\right] d x^{5} .
$$

The phantom field is related with the dilaton field $\Phi$ via $\Phi-\Phi_{0}=i\left(\Phi_{p}-\Phi_{p_{0}}\right)$, and the potential for $a<1$ becomes

$$
\tilde{V}\left(\Phi_{p}\right)=-\frac{12}{L^{2}}+\frac{3 a(3 a+1)}{L^{2 a}} \exp \left( \pm \frac{4}{3} \sqrt{\frac{1-a}{a}}\left(\Phi_{p}-\Phi_{p_{0}}\right)\right)
$$

Instead of relations (6),(7) for the phantom field one gets

$$
\partial_{z} \Phi_{p}= \pm \frac{3}{2} \sqrt{\left(\frac{b^{\prime \prime}}{b}-\frac{2\left(b^{\prime}\right)^{2}}{b^{2}}\right)}, \quad \tilde{V}\left(\Phi_{p}(z)\right)=\frac{3}{b^{2}}\left(\frac{b^{\prime \prime}}{b}+\frac{2\left(b^{\prime}\right)^{2}}{b^{2}}-\frac{4 b^{2}}{L^{2}}\right) .
$$

2.1.3 Mixed factor of the form $b(z)=\left(\frac{L}{z}\right)^{a} \exp \left(-z^{2} / R^{2}\right)$

Now we consider a space with the mixed $b$-factor of the form

$$
b(z)=\left(\frac{L}{z}\right)^{a} \exp \left(-z^{2} / R^{2}\right)
$$


The superpotentials method cannot be applied to this case, since $z$-variable cannot be represented through a new $u$-variable explicitly and we cannot represent $b(u)$ explicitly. For this $b$-factor we can express $\partial_{z} \Phi(z)$ and $V(\Phi(z))$ using (6) , (17) as

$$
\begin{aligned}
& \partial_{z} \Phi(z)= \pm \frac{3}{2} \frac{\xi}{R^{2} z}, \text { where } \quad \xi=\sqrt{\zeta}, \quad \zeta=4 z^{4}+2 R^{2}(2 a+1) z^{2}+a R^{4}(a-1), \\
& V(z)=-\frac{12}{L^{2}}+\frac{3\left(\frac{L}{z}\right)^{-2 a}\left(a R^{4}(3 a+1)+2 z^{2} R^{2}(6 a-1)+12 z^{4}\right) \exp \left(\frac{2 z^{2}}{R^{2}}\right)}{z^{2} R^{4}} .
\end{aligned}
$$

Integrating (17) we get

$$
\begin{gathered}
\Phi_{ \pm}= \pm\left(\frac{3}{4} \frac{\xi}{R^{2}}+\frac{3}{8}(2 a+1) \ln \left(\xi+\frac{(2 a+1) R^{2}+4 z^{2}}{2}\right)-\right. \\
\left.\frac{3}{4} \sqrt{a(a-1)} \ln \left(\frac{2 R^{2}\left\{a(a-1) R^{2}+(2 a+1) z^{2}+\xi \sqrt{a(a-1)}\right\}}{z^{2}}\right)\right)+\Phi_{0 \pm} .
\end{gathered}
$$

For $a>1$ all expressions in (19) are well defined. We can find $V(\Phi)$ at $z \rightarrow \infty, \Phi \sim \frac{3}{2} \frac{z^{2}}{R^{2}}$ and $V \sim \Phi^{a+1} e^{\frac{4}{3} \Phi}$.

We present $V(\Phi)$ graphically in Fig 1 for $a=1, a=2$.

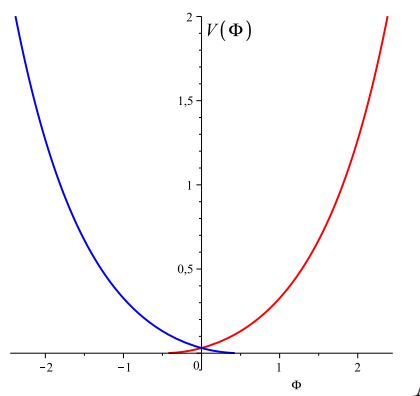

A.

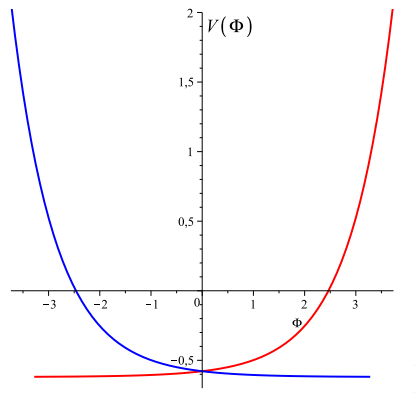

B.

Figure 1: The potentials corresponding to $b(z)$ in the form (16) at $a=2, L=4.4 \mathrm{fm}, R=1$ fm (the left panel) and at $a=1, L=4.4 \mathrm{fm}, R=1 \mathrm{fm}$ (the right panel) and $\Phi_{0}=-0.5$. Two branches, red and blue, correspond to \pm in (17) (the red for + , the blue for - ).

For $a<1, \zeta(z)$ has a positive root, $\zeta\left(z_{0}\right)=0$, and $\zeta(z)>0$ for $z>z_{0}, \zeta(z)<0$ for $0<z<z_{0}$, see Fig 2. For example, in the case of $a=1 / 3$, the point $z_{0} \approx 0.249 \mathrm{fm}$ and for $a=1 / 2$, the point $z_{0} \approx 0.243 \mathrm{fm}$.

Near $z=z_{0}$ the function $\Phi(z)$ has a singularity of the form

$$
\Phi(z) \sim\left(z^{2}-z_{0}^{2}\right)^{3 / 2}\left(A+\mathcal{O}\left(z^{2}-z_{0}^{2}\right)\right), \quad A= \pm \frac{2(8 a+1)^{1 / 4}}{\sqrt{z_{0}}},
$$

$\Phi(z)$ is real for $z>z_{0}$,

$$
\Phi_{ \pm}=\frac{3}{4 R^{2}}\left(\sqrt{\zeta}-\sqrt{-\left(a^{2}-a\right) R^{4}} \arctan \left(\frac{\left(a^{2}-a\right) R^{4}+(2 a+1) R^{2} z^{2}}{\sqrt{\zeta} \sqrt{-\left(a^{2}-a\right) R^{4}}}\right)+\right.
$$




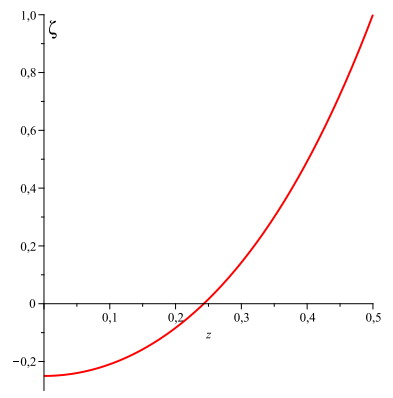

Figure 2: The function $\zeta=\zeta(z)$ at $a=1 / 2, R=1 \mathrm{fm}$.

$$
\left.\frac{1}{2}(2 a+1) R^{2} \ln \left(a\left(4 \sqrt{\zeta}+8 z^{2}+2(2 a+1) R^{2}\right)\right)\right)+\Phi_{0 \pm}, \quad z>z_{0}
$$

and becomes imaginary for $z<z_{0},\left(\Phi_{ \pm}-\Phi_{0 \pm}\right)=i\left(\Phi_{p \pm}-\Phi_{p 0 \pm}\right)$,

$$
\begin{aligned}
\Phi_{p \pm}= \pm & \frac{3}{4 R^{2}}\left(\sqrt{a R^{4}(1-a)} \ln \left(\frac{2 \sqrt{a R^{4}(1-a)} \sqrt{-\zeta}+2 R^{2}\left[\left(a-a^{2}\right) R^{2}-2 z^{2}(a+1 / 2)\right]}{z^{2}}\right)\right. \\
& \left.-\left(\left(a+\frac{1}{2}\right) R^{2} \arctan \left(\frac{(2 a+1) R^{2}+4 z^{2}}{2 \sqrt{-\zeta}}\right)-\sqrt{-\zeta}\right)\right)+\Phi_{0 \pm}, \quad z<z_{0} .
\end{aligned}
$$

The convenient choice of constants is $\Phi_{s \pm}\left(z_{0}\right)=\Phi_{p \pm}\left(z_{0}\right)=0$. The imaginary scalar field corresponds to the phantom sign of the kinetic term and one can write $\Phi=\Phi_{s} \Theta\left(z-z_{0}\right)+$ $i \Phi_{p} \Theta\left(z_{0}-z\right)$ and interpret this model as a model with an alternating sign of the kinetic term.

The potential can be represented parametrically as a functions of the real component $\Phi_{s}$ for $z>z_{0}$ and as a function of the imaginary component $\Phi_{p}$ for $z<z_{0}$, see Fig. 3 ,

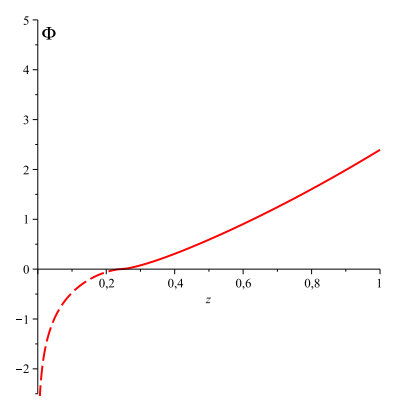

A.
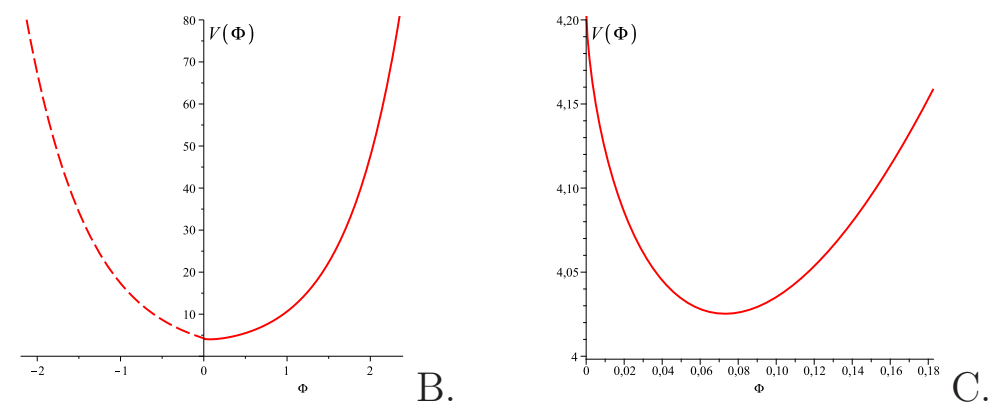

Figure 3: The plots corresponding to $a=1 / 2, L=4.4 \mathrm{fm}, \mathrm{R}=1 \mathrm{fm}$ and a sign plus in (17). A. The phantom $\Phi_{p}$ (dashed line) and dilaton $\Phi_{s}$ (solid line) fields as functions of $z$. B. The dependence of the potential $V$ on the dilaton and the phantom fields. C. The same dependence of the potential $V$ on the dilaton field as in B for small $\Phi$. 


\subsection{Shock wave}

To deal with a point-like shock wave we add to the action (11) an action of a point-like source moving along a trajectory $x^{\mu}=x_{*}^{\mu}(\eta)$,

$$
S_{\mathrm{st}}=\int\left[\frac{1}{2 e} g_{\mu \nu} \frac{d x_{*}^{\mu}}{d \eta} \frac{d x_{*}^{\nu}}{d \eta}-\frac{e}{2} m^{2}\right] d \eta
$$

$m$ is the particle mass, $\eta$ is an arbitrary world-line parameter, we assume that the particle mass is zero, which allows treating only with light-like geodesics. $e_{\mu}^{a}$ is the frame associated with the metric, $g_{\mu \nu}=e_{\mu}^{a} e_{\nu a}$, and $e$ is the square root of its determinant $e=\sqrt{-g}$. We assume that the metric has the shock wave form [24]-28]

$$
d s^{2}=b^{2}(z)\left(d z^{2}+d x^{i} d x^{i}-d x^{+} d x^{-}+\phi\left(z, x^{1}, x^{2}\right) \delta\left(x^{+}\right)\left(d x^{+}\right)^{2}\right), \quad i=1,2 .
$$

The shock-wave metric solves the Einstein equation

$$
\left(R_{\mu \nu}-\frac{g_{\mu \nu}}{2} R\right)-\frac{g_{\mu \nu}}{2}\left(-\frac{4}{3}(\partial \Phi)^{2}+V(\Phi)\right)-\frac{4}{3} \partial_{\mu} \Phi \partial_{\nu} \Phi-g_{\mu \nu} \frac{d(d-1)}{2 L^{2}}=8 \pi G_{5} J_{\mu \nu}
$$

where $(\partial \Phi)^{2}=g^{\mu \nu} \partial_{\mu} \Phi \partial_{\nu} \Phi$, and the current $J_{\mu \nu}$ is given by [6]

$$
J_{\mu \nu}=\frac{1}{\sqrt{-g}} \int e p_{\mu} p_{\nu} \delta\left(x^{\mu}-x_{*}^{\mu}\right) d \eta
$$

here $p_{\mu}$ is the conjugate momentum

$$
p_{\mu}=e^{-1} g_{\mu \nu} \frac{d x^{\nu}}{d \eta}
$$

The current in light-like coordinates $\left(x^{+}, x^{-}, x^{i}, z\right), i=1,2$ is written by

$$
J_{++}=\frac{E}{b^{3}(z)} \delta\left(x^{1}\right) \delta\left(x^{2}\right) \delta\left(z-z_{*}\right) \delta\left(x^{+}\right) .
$$

As the metric (21) has the addition of the shock wave profile in comparison with the metric (2) , so we have the additional Einstein equation describing the shock wave profile $\phi\left(z, x_{\perp}\right)$

$$
\left(\partial_{x^{1}}^{2}+\partial_{x^{2}}^{2}+\partial_{z}^{2}+\frac{3 b^{\prime}}{b} \partial_{z}\right) \phi\left(z, x_{\perp}\right)=-16 \pi G_{5} \frac{E}{b^{3}} \delta\left(x^{1}\right) \delta\left(x^{2}\right) \delta\left(z-z_{*}\right) .
$$

Hence, it is clear that the dilaton field does not explicitly affect on the shock wave profile resulting from the source.

\subsection{Domain-wall}

Here, we consider the Einstein equations for the shock wave resulting from mass uniformly distributed over the domain-wall. The shock wave motion generated by a point mass corresponds to equation (23). To obtain the Einstein equations for the shock waves generated by a domain-wall, we consider the mass of a point-like source averaged over the domain-wall. Such an averaging method was proposed in [10], and we considered it in [13]. To derive the 
equations of the domain-wall, we use the expression for the induced metric over the wall surface:

$$
h_{\alpha \beta}=\frac{\partial x^{\mu}}{\partial \sigma^{\alpha}} \frac{\partial x^{\nu}}{\partial \sigma^{\beta}} g_{\mu \nu}=b^{2} \delta_{\alpha \beta} .
$$

We integrate (23) over $x_{\perp}=\left(x_{1}, x_{2}\right)$. According to (24), one has

$$
\int \sqrt{h} d x_{\perp}=\int b^{2} d x_{\perp}
$$

and hence

$$
\int b^{2}\left(\partial_{z}^{2}+\partial_{x^{1}}^{2}+\partial_{x^{2}}^{2}+\frac{3 b^{\prime}}{b} \partial_{z}\right) \phi\left(z, x_{\perp}\right) d x_{\perp}=-16 \pi G_{5} b^{2} \frac{E}{b^{3}} \delta\left(z-z_{*}\right) .
$$

Assuming that the derivatives of $\phi\left(z, x_{\perp}\right)$ with respect to the transversal variables $x_{\perp}$ to be decrease at $\pm \infty$, we obtain the equation of motion for the membrane wall:

$$
\left(\partial_{z}^{2}+\frac{3 b^{\prime}}{b} \partial_{z}\right) \phi^{\mathrm{W}}(z)=-16 \pi G_{5} \frac{E}{b^{3}} \delta\left(z-z_{*}\right),
$$

where

$$
\phi^{\mathrm{W}}(z)=\int \phi\left(z, x_{\perp}\right) d x_{\perp} .
$$

We can assume the size of the moving domain is finite and average the mass over the finite surface in (23). We consider a wave profile which is provided by the mass uniformly distributed over the surface perpendicular to the direction of motion. Therefore, wave profile (25) depends on the coordinate along which the motion occurs, and the equation of the wave profile becomes

$$
\left(\partial_{z}^{2}+\frac{3 b^{\prime}}{b} \partial_{z}\right) \phi^{\omega}(z)=-16 \pi G_{5} \frac{E}{b^{3}} \delta\left(x_{\perp}\right) \delta\left(z-z_{*}\right) .
$$

The assumption about the domain is a disk of radius $L$ allows to transform the equation into the form

$$
\left(\partial_{z}^{2}+\frac{3 b^{\prime}}{b} \partial_{z}\right) \phi^{\omega}(z)=-16 \pi G_{5} \frac{E^{*}}{b^{3}} \delta\left(z-z_{*}\right), \quad E^{*}=\frac{E}{L^{2}}
$$

This shows that the cases of the mass distribution over finite and infinite surfaces are equivalent, i. e. the profiles are differed by a constant factor corresponding to the size of the finite object $\phi^{\omega}(z)=\phi^{\mathrm{W}}(z) / L^{2}$.

\subsection{Condition of the trapped surface formation}

In the case of $b=L / z$, the conditions on the boundary points $z_{a}, z_{b}$ of the trapped surface were obtained in [10, 13]:

$$
\left.\left(\partial_{z} \phi^{\omega}\right)\right|_{z=z_{a}}=2,\left.\quad\left(\partial_{z} \phi^{\omega}\right)\right|_{z=z_{b}}=-2
$$


where $z_{a}<z_{*}<z_{b}$ is assumed. Obviously, expressions (26) lead to the condition 2 $\left.\left(\partial_{z} \phi^{\omega}\right)^{2}\right|_{\mathrm{TS}}=4$. Due to $\phi^{W}(z)=L^{2} \phi^{\omega}(z)$, the condition on the boundary of the trapped surface formed by the collision of two infinity domain-walls is written as

$$
\left.\left(\partial_{z} \phi^{\mathrm{W}}\right)^{2}\right|_{\mathrm{TS}}=4 L^{4}
$$

It is therefore obvious that the values of boundary points of the trapped surface are independent of whether the mass is distributed over a finite or infinite surface.

\section{Power-law b-factor}

We note that the power-law factor of the form $b=\left(L /\left(z-z_{0}\right)\right)^{a}$ was used in [14]. If $a=1$ and $b=L /\left(z-z_{0}\right)$, then the Einstein equation becomes

$$
\left(\partial_{z}^{2}-\frac{3}{z-z_{0}} \partial_{z}\right) \phi^{\omega}(z)=-16 \pi G_{5} E^{*} \frac{\left(z-z_{0}\right)^{3}}{L^{3}} \delta\left(z-z_{*}\right) .
$$

Because $z_{0}$ is a constant, replacing $z$ with $z-z_{0}$ reduces Eq. (27) to the equation considered in [10]. For definiteness in what follows, we assume that $z_{0}=0$ in the power-law $b$-factors.

The equation of the domain-wall profile in the space with the power-law factor $b=(L / z)^{a}$ is written as

$$
\left(\partial_{z}^{2}-\frac{3 a}{z} \partial_{z}\right) \phi^{\omega}(z)=-16 \pi G_{5}\left(\frac{z}{L}\right)^{3 a} E^{*} \delta\left(z-z_{*}\right) .
$$

We consider this equation separately before and after the collision. The boundary points of trapped surface of the black hole are denoted by $z_{a}$ and $z_{b}, z_{a}<z_{*}<z_{b}$. The solution of (28) is written as

$$
\phi^{\omega}(z)=\phi_{a}^{\omega} \Theta\left(z_{*}-z\right)+\phi_{b}^{\omega} \Theta\left(z-z_{*}\right)
$$

where

$$
\begin{aligned}
& \phi_{a}^{\omega}(z)=C_{0} z_{a} z_{b}\left(\left(\frac{z_{*}}{z_{b}}\right)^{3 a+1}-1\right)\left(\left(\frac{z}{z_{a}}\right)^{3 a+1}-1\right), \\
& \phi_{b}^{\omega}(z)=C_{0} z_{a} z_{b}\left(\left(\frac{z_{*}}{z_{a}}\right)^{3 a+1}-1\right)\left(\left(\frac{z}{z_{b}}\right)^{3 a+1}-1\right), \\
& C_{0}=-\frac{16 \pi G_{5} E z_{a}^{3 a} z_{b}^{3 a}}{(1+3 a) L^{3 a+2}\left(z_{b}^{3 a+1}-z_{a}^{3 a+1}\right)} .
\end{aligned}
$$

The given profile is presented in Figs. 4, 5 .

\footnotetext{
${ }^{2}$ We note that the condition $\left.\left(\partial_{z} \phi^{\omega}\right)^{2}\right|_{\text {TS }}=8$ was used in [14. The difference in the boundary conditions is associated with the choice of the shock wave metric in [14] in the form

$$
d s^{2}=b^{2}\left\{d z^{2}+d x^{i} d x^{i}-2 d x^{+} d x^{-}+\phi\left(z, x^{1}, x^{2}\right)\left(d x^{+}\right)^{2}\right\}
$$
}




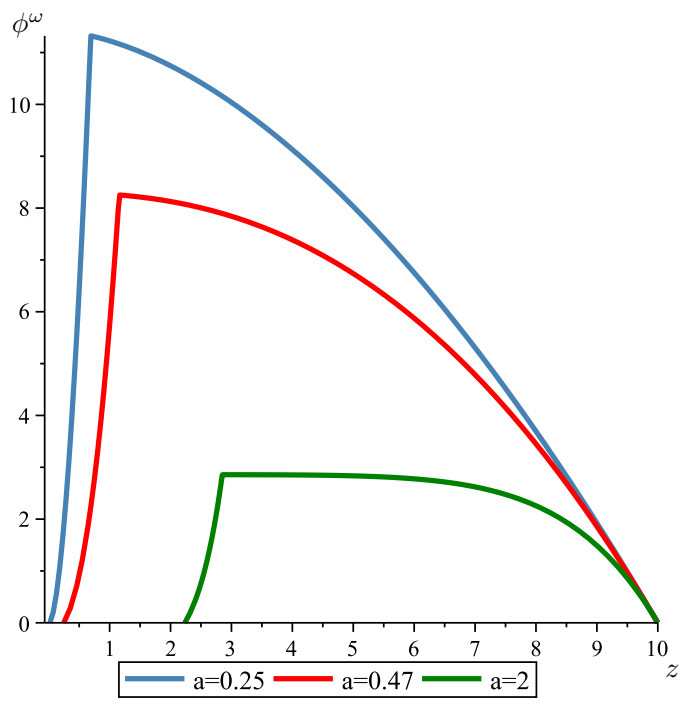

Figure 4: The profiles $\phi^{\omega}(z)$ corresponding to $b=\left(\frac{L}{z}\right)^{a}$ at $a=0.25, a=0.47, a=2$, $z_{b}=10 \mathrm{fm}, E=0.2 \mathrm{GeV}$. The unit of length in Figs. 412, 14] is $1 \mathrm{fm}$.

For profile (29), the formation conditions for the trapped surface at the boundary points $z=z_{a}$ and $z=z_{b}$ are

$$
\begin{aligned}
& \frac{8 \pi G_{5} E z_{a}^{3 a}\left(1-z_{b}^{3 a+1} / z_{*}^{3 a+1}\right)}{L^{3 a+2}\left(z_{b}^{3 a+1} / z_{*}^{3 a+1}-z_{a}^{3 a+1} / z_{*}^{3 a+1}\right)}=-1, \\
& \frac{8 \pi G_{5} E z_{b}^{3 a}\left(1-z_{a}^{3 a+1} / z_{*}^{3 a+1}\right)}{L^{3 a+2}\left(z_{b}^{3 a+1} / z_{*}^{3 a+1}-z_{a}^{3 a+1} / z_{*}^{3 a+1}\right)}=1 .
\end{aligned}
$$

The collision point $z_{*}$ can not be fixed but found from the system regarded as a system of equations for $z_{*}, z_{a}$ with a given $z_{b}$ :

$$
z_{a}=\left(\frac{z_{b}^{3 a}}{-1+z_{b}^{3 a} C}\right)^{1 / 3 a}, \quad z_{*}=\left(\frac{z_{a}^{3 a} z_{b}^{3 a}\left(z_{b}+z_{a}\right)}{z_{a}^{3 a}+z_{b}^{3 a}}\right)^{1 /(3 a+1)},
$$

where $C=8 \pi G_{5} E / L^{3 a+2}$. The solution of this system is shown in Fig. 6.

As a consequence of the condition $z_{a}<z_{b}$ and (31), we obtain the range of the energies: $E>L^{3 a+2} / 4 \pi G_{5} z_{b}^{3 a}$ at which the black hole profile is created. It is clear that the black hole profile is not created at the energy $E=L^{3 a+2} / 4 \pi G_{5} z_{b}^{3 a}$, and for the parameters $G_{5}=L^{3} / 1.9$, $L=4.4 \mathrm{fm}$ and $z_{b}=0.5 \mathrm{fm}$ it equals to $E=3.71 \mathrm{MeV}$. (see. Fig. 5).

For $z_{b}^{3 a} C \gg 1$, we consider $z_{a} \ll z_{*} \ll z_{b}$. Based on (31), we have the approximation:

$$
z_{a} \sim\left(\frac{1}{C}\right)^{1 / 3 a}, \quad z_{*} \sim\left(\frac{z_{b}}{C}\right)^{1 /(3 a+1)} .
$$

The trapped surface area is calculated as

$$
S_{\text {trap }}=\frac{1}{2 G_{5}} \int_{C} \sqrt{\operatorname{det}\left|g_{\mathrm{AdS}_{3}}\right|} d z d^{2} x_{\perp}
$$




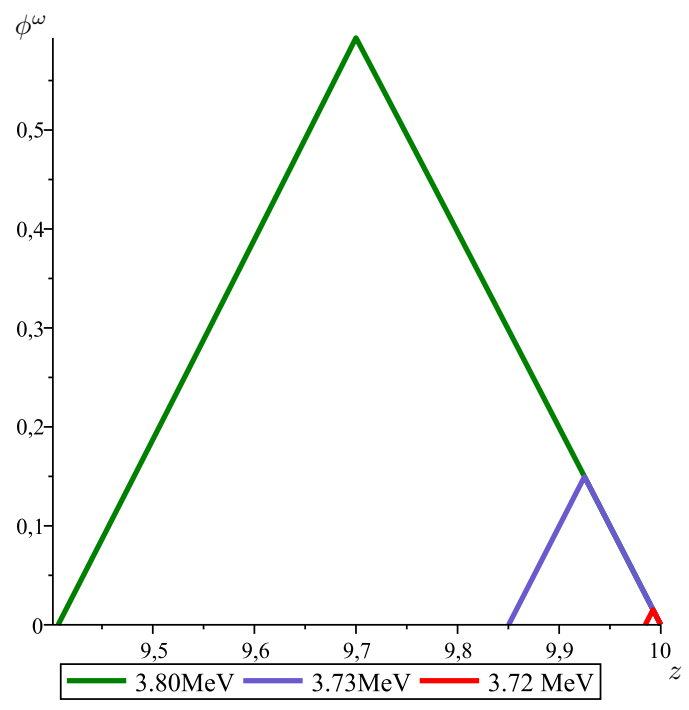

Figure 5: The profiles $\phi^{\omega}(z)$ corresponding to $b=\left(\frac{L}{z}\right)^{a}$ at $a=0.25, z_{b}=10 \mathrm{fm}, E=3.72$ $\mathrm{MeV}, E=3.73 \mathrm{MeV}, E=3.80 \mathrm{MeV}$.

where det $\left|g_{\mathrm{AdS}_{3}}\right|$ is the metric determinant of free dimensional space $\mathrm{AdS}_{3}$. In the follows, we calculate the relative area $s$ of the trapped surface defined by

$$
s=\frac{S_{\text {trap }}}{\int d^{2} x_{\perp}}=\frac{1}{2 G_{5}} \int_{z_{a}}^{z_{b}} b^{3} d z .
$$

In the considered case where $b(z)=(L / z)^{a}$, the formula for the relative area of trapped surface becomes

$$
s=\frac{1}{2 G_{5}(3 a-1)}\left(z_{a}\left(\frac{L}{z_{a}}\right)^{3 a}-z_{b}\left(\frac{L}{z_{b}}\right)^{3 a}\right),
$$

and $s$ determines the relative entropy. With the assumption $3 a>1$ and the used approximation, it is clear from this expression that the relative area of the trapped surface tends to its maximum value at infinite $z_{b}$ :

$$
\left.s\right|_{z_{b} \rightarrow \infty}=\frac{L^{3 a}}{2 G_{5}(3 a-1)} z_{a}^{1-3 a}=\frac{L}{2 G_{5}}\left(\frac{8 \pi G_{5}}{L^{2}}\right)^{(3 a-1) / 3 a} E^{(3 a-1) / 3 a} .
$$

We thus find that for $a>1 / 3$, the entropy $S$ increases as $E^{(3 a-1) / 3 a}$.

We substitute parameters and variables with the dimension of length in formula (32) using the relation $1 \mathrm{GeV} \approx 5 \mathrm{fm}^{-1}$ and choose the parameters $G_{5}$ and $L$ based on phenomenological reasons [6]: $G_{5}=L^{3} / 1.9$ and $L=4.4 \mathrm{fm}$. We here assume that we consider collisions of lead ions. The multiplicity of particles produced in heavy-ion collisions ( $\mathrm{Pb}-\mathrm{Pb}$ and $\mathrm{Au}-\mathrm{Au}$ collisions) depends on energy as $s_{N N}^{0.15}$ according to the experimental data [19] in the range from 10 to $10^{3} \mathrm{GeV}$. Therefore, $a \approx 0.47$. For $a=0.47$, we have

$$
\frac{L}{2 G_{5}}\left(\frac{8 \pi G_{5}}{L^{2}}\right)^{(3 a-1) / 3 a} \approx 0.16 \mathrm{fm}^{-1.85}
$$




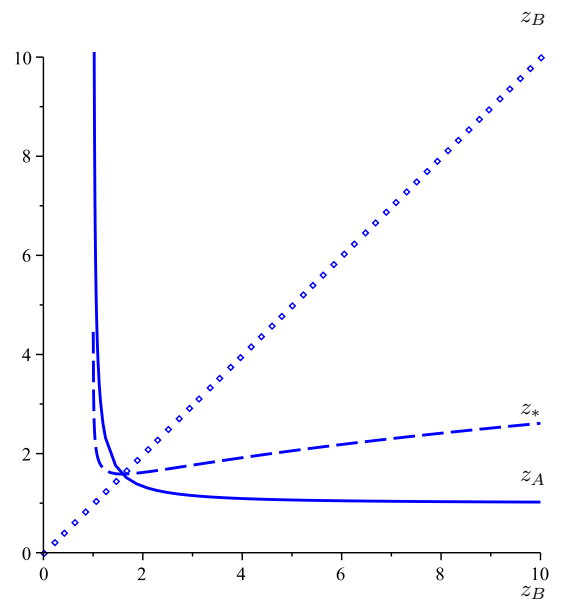

Figure 6: The solution to the system of equations (30) for the given $z_{b}$.

\section{Factor of the form $b=\mathrm{e}^{-z / R}$}

The equation of the domain-wall wave profile in the space with the exponential $b$-factor $b=\mathrm{e}^{-z / R}$ is written as

$$
\left(\partial_{z}^{2}-\frac{3}{R} \partial_{z}\right) \phi^{\omega}(z)=-16 \pi G_{5} E^{*} \mathrm{e}^{3 z / R} \delta\left(z-z_{*}\right)
$$

and we construct the solution in the form

$$
\phi^{\omega}(z)=\phi_{a}(z) \Theta\left(z_{*}-z\right)+\phi_{b}(z) \Theta\left(z-z_{*}\right)
$$

where

$$
\begin{aligned}
\phi_{a} & =C_{a} \frac{R}{3} \mathrm{e}^{3 z / R}+\widetilde{C}_{a}, & \phi_{b} & =C_{b} \frac{R}{3} \mathrm{e}^{3 z / R}+\widetilde{C}_{b}, \\
C_{a} & =-\frac{16 \pi G_{5} E^{*}\left(\mathrm{e}^{3 z_{*} / R}-\mathrm{e}^{3 z_{b} / R}\right)}{\mathrm{e}^{3 z_{b} / R}-\mathrm{e}^{3 z_{a} / R}}, & C_{b} & =-\frac{16 \pi G_{5} E^{*}\left(\mathrm{e}^{3 z_{*} / R}-\mathrm{e}^{3 z_{a} / R}\right)}{\mathrm{e}^{3 z_{b} / R}-\mathrm{e}^{3 z_{a} / R}}, \\
\widetilde{C}_{a} & =-C_{a} \frac{R}{3} \mathrm{e}^{3 z_{a} / R}, & \widetilde{C}_{b} & =-C_{b} \frac{R}{3} \mathrm{e}^{3 z_{b} / R} .
\end{aligned}
$$

The conditions at the boundaries of trapped surface are

$$
\begin{aligned}
& \frac{8 \pi G_{5} E}{L^{2}} \frac{\left(\mathrm{e}^{3 z_{*} / R}-\mathrm{e}^{3 z_{b} / R}\right) \mathrm{e}^{3 z_{a} / R}}{\mathrm{e}^{3 z_{b} / R}-\mathrm{e}^{3 z_{a} / R}}=-1, \\
& \frac{8 \pi G_{5} E}{L^{2}} \frac{\left(\mathrm{e}^{3 z_{*} / R}-\mathrm{e}^{3 z_{a} / R}\right) \mathrm{e}^{3 z_{b} / R}}{\mathrm{e}^{3 z_{b} / R}-\mathrm{e}^{3 z_{a} / R}}=1 .
\end{aligned}
$$

We analyze these conditions. We set

$$
Z_{*}=\mathrm{e}^{3 z_{*} / R}, \quad Z_{a}=\mathrm{e}^{3 z_{a} / R}, \quad Z_{b}=\mathrm{e}^{3 z_{b} / R} .
$$

and substitute these values in conditions (33). We obtain the equations

$$
\frac{8 \pi G_{5} E}{L^{2}} \frac{\left(Z_{*}-Z_{b}\right) Z_{a}}{Z_{b}-Z_{a}}=-1, \quad \frac{8 \pi G_{5} E}{L^{2}} \frac{\left(Z_{*}-Z_{a}\right) Z_{b}}{Z_{b}-Z_{a}}=1 .
$$



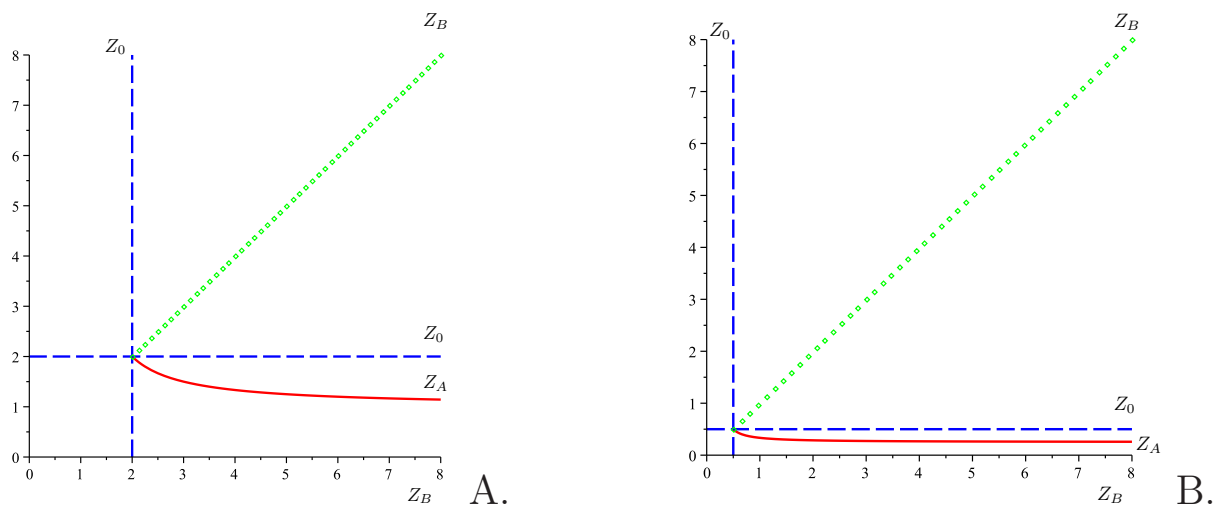

Figure 7: Solution (35) at $Z_{*}=4$ and (b) $Z_{*}=2$ : the vertical dashed line separates the region where $Z_{b}>Z_{*}$.

As in the previous case, we consider the equations for $Z_{a}$ and $Z_{*}$ with a fixed $Z_{b}$.

This system has the trivial solution $Z_{a}=Z_{b}=Z_{*}$ and the solution

$$
Z_{a}=\frac{L^{2}}{8 \pi G_{5} E} \frac{Z_{b}}{Z_{b}-L^{2} / 8 \pi G_{5} E}, \quad Z_{*}=\frac{L^{2}}{4 \pi G_{5} E} .
$$

This solution is shown in Fig. 7 at two values of the parameter $Z_{*}$ :

$$
\frac{Z_{*}}{4}=1, \quad \frac{Z_{*}}{4}=\frac{1}{2} .
$$

The relative area of trapped surface is given by

$$
s=\frac{R}{6 G_{5}}\left(\frac{1}{\mathrm{e}^{3 z_{a} / R}}-\frac{1}{\mathrm{e}^{3 z_{b} / R}}\right)=\frac{R}{6 G_{5}}\left(\frac{1}{Z_{a}}-\frac{1}{Z_{b}}\right) .
$$

The maximum entropy is attained for $Z_{b} \gg 1$. In this approximation,

$$
Z_{a} \sim \frac{L^{2}}{8 \pi G_{5} E}, \quad s \sim \frac{4}{3} \frac{\pi E R}{L^{2}} .
$$

According to (35) and (34) we get the colliding point and the boundary one $z_{a}$, namely,

$$
\begin{gathered}
z_{*}=\frac{R}{3} \ln \left(\frac{L^{2}}{4 \pi E G_{5}}\right), \\
z_{a}=\frac{R}{3} \ln \left(\frac{e^{3 z_{b} / R}}{\frac{8 \pi G_{5} E}{L^{2}} e^{3 z_{b} / R}-1}\right) .
\end{gathered}
$$

Based on the condition that $z_{a}<z_{b}$, the lowest energy limit is found, and due to the condition $z_{a}>0$, the highest energy limit is defined, so

$$
\frac{L^{2}}{4 \pi G_{5}} e^{-3 z_{b} / R}<E<\frac{L^{2}}{8 \pi G_{5}}\left(1+e^{-3 z_{b} / R}\right) .
$$

For the parameters $G_{5}=L^{3} / 1.9, L=4.4 \mathrm{fm}$ and $z_{b}=0.5 \mathrm{fm}$ it takes the values

$$
1.53 \mathrm{MeV}<E<4.20 \mathrm{MeV} .
$$

The corresponding profile $\phi^{\omega}$ is represented in Fig. 8 . 


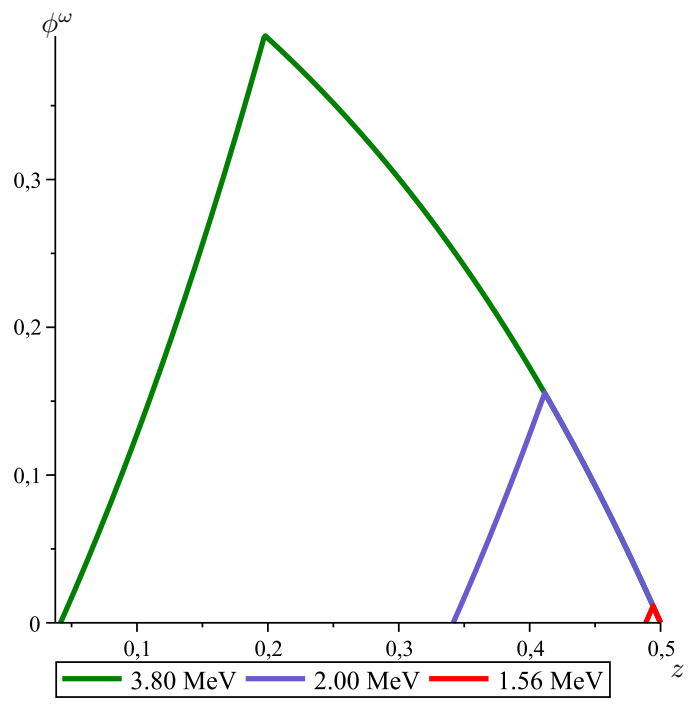

Figure 8: The profiles $\phi^{w}(z)$ corresponding to $b=\mathrm{e}^{-z / R}$ at $z_{b}=0.5 \mathrm{fm}, R=1 \mathrm{fm}, E=1.56$ $\mathrm{MeV}, E=2.00 \mathrm{MeV}, E=3.80 \mathrm{MeV}$.

\section{$5 \quad$ Mixed factor of the form $b=(L / z) \mathrm{e}^{-z^{2} / R^{2}}$}

The equation of the profile corresponding to the domain-wall motion in the space with $b$-factor of the form $b=(L / z) \mathrm{e}^{-z^{2} / R^{2}}$ is written as

$$
\left(\partial_{z}^{2}-3\left(\frac{1}{z}+\frac{2 z}{R^{2}}\right) \partial_{z}\right) \phi^{\omega}=-16 \pi G_{5} E^{*}\left(\frac{z}{L}\right)^{3} \mathrm{e}^{3 z^{2} / R^{2}} \delta\left(z-z_{*}\right) .
$$

We consider the solution of the obtained equation:

$$
\phi^{\omega}=\phi_{a}^{\omega} \Theta\left(z_{*}-z\right)+\phi_{b}^{\omega} \Theta\left(z-z_{*}\right),
$$

where

$$
\begin{aligned}
& \phi_{a}^{\omega}=-C_{a}\left(R^{2}-3 z_{a}^{2}\right) \mathrm{e}^{3 z_{a}^{2} / R^{2}}+C_{a}\left(R^{2}-3 z^{2}\right) \mathrm{e}^{3 z^{2} / R^{2}}, \\
& \phi_{b}^{\omega}=-C_{b}\left(R^{2}-3 z_{b}^{2}\right) \mathrm{e}^{3 z_{b}^{2} / R^{2}}+C_{b}\left(R^{2}-3 z^{2}\right) \mathrm{e}^{3 z^{2} / R^{2}},
\end{aligned}
$$

and the factors $C_{a}$ and $C_{b}$ are given by

$$
\begin{aligned}
& C_{a}=C_{0}\left(-\left(R^{2}-3 z_{b}^{2}\right) \mathrm{e}^{3 z_{b}^{2} / R^{2}}+\left(R^{2}-3 z_{*}^{2}\right) \mathrm{e}^{3 z_{*}^{2} / R^{2}}\right), \\
& C_{b}=C_{0}\left(-\left(R^{2}-3 z_{a}^{2}\right) \mathrm{e}^{3 z_{a}^{2} / R^{2}}+\left(R^{2}-3 z_{*}^{2}\right) \mathrm{e}^{3 z_{*}^{2} / R^{2}}\right),
\end{aligned}
$$

where

$$
C_{0}=\frac{8 \pi G_{5} E^{*} R^{2}}{9 L^{3}\left(\left(R^{2}-3 z_{b}^{2}\right) \mathrm{e}^{3 z_{b}^{2} / R^{2}}-\left(R^{2}-3 z_{a}^{2}\right) \mathrm{e}^{3 z_{a}^{2} / R^{2}}\right)} .
$$

Differentiating each of the two terms in the right-hand sides of solution (40), we get

$$
\frac{d \phi_{a}^{\omega}(z)}{d z}=-C_{a} \frac{18 z^{3}}{R^{2}} \mathrm{e}^{3 z^{2} / R^{2}}, \quad \frac{d \phi_{b}^{\omega}(z)}{d z}=-C_{b} \frac{18 z^{3}}{R^{2}} \mathrm{e}^{3 z^{2} / R^{2}}
$$



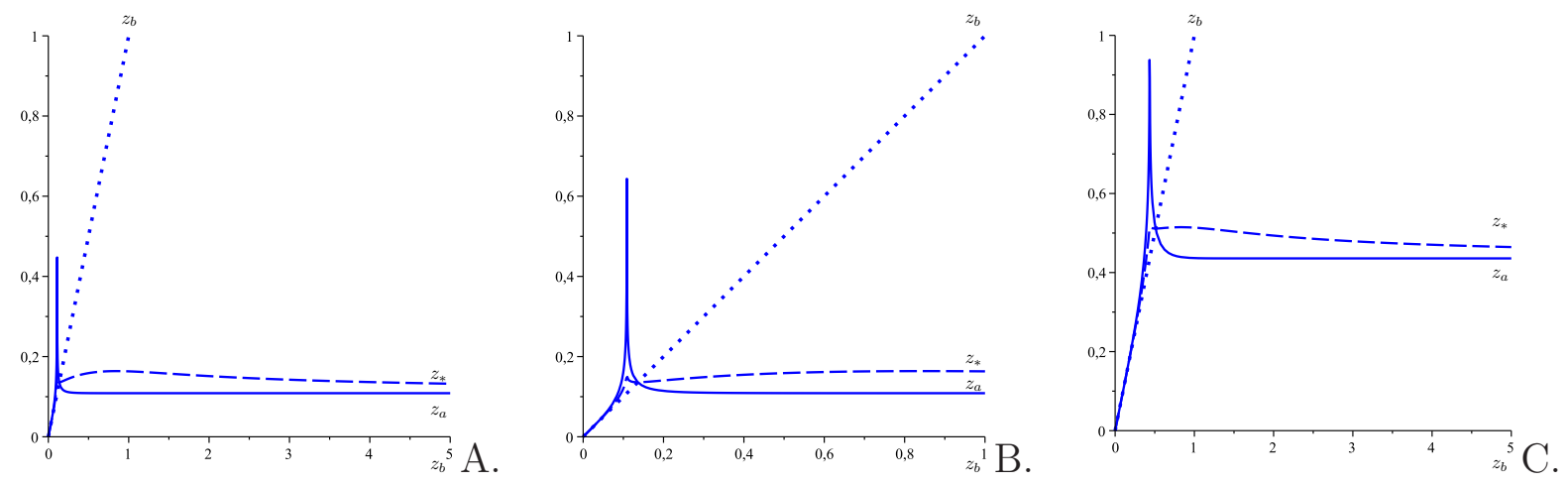

Figure 9: The dependence $z_{a}, z_{*}$ on $z_{b}$ at energies $E=220 \mathrm{GeV}$ (Fig. A. at $z_{b} \leq 5 \mathrm{fm}$, Fig. B. at $z_{b} \leq 1 \mathrm{fm}$ ) and $E=2 \mathrm{GeV}$ ( Fig. C.).

Hence, the conditions for the trapped surface formation at the boundary points become

$$
C_{a} \frac{9 z_{a}^{3}}{R^{2}} \mathrm{e}^{3 z_{a}^{2} / R^{2}}=-1, \quad C_{b} \frac{9 z_{b}^{3}}{R^{2}} \mathrm{e}^{3 z_{b}^{2} / R^{2}}=1 .
$$

Considering this system with $C_{a}$ and $C_{b}$ defined by formulas (41) and (42), we obtain a system of two equations for the three unknowns $z_{a}, z_{b}$ and $z_{*}$. We assume that $z_{a}$ and $z_{*}$ are unknown and $z_{b}$ is given.

From system of equations (43), we can obtain the relations

$$
\begin{aligned}
& z_{a}^{3} \mathrm{e}^{3 z_{a}^{2} / R^{2}}=\frac{L^{3} z_{b}^{3} \mathrm{e}^{3 z_{b}^{2} / R^{2}}}{8 \pi G_{5} E^{*} z_{b}^{3} \mathrm{e}^{3 z_{b}^{2} / R^{2}}-L^{3}}, \\
& \left(R^{2}-3 z_{*}^{2}\right) \mathrm{e}^{3 z_{*}^{2} / R^{2}}=\frac{\left(z_{a}^{3} R^{2}-3 z_{a}^{3} z_{b}^{2}-3 z_{b}^{3} z_{a}^{2}+z_{b}^{3} R^{2}\right) \mathrm{e}^{3 z_{a}^{2} / R^{2}} \mathrm{e}^{3 z_{b}^{2} / R^{2}}}{z_{a}^{3} \mathrm{e}^{3 z_{a}^{2} / R^{2}}+z_{b}^{3} \mathrm{e}^{3 z_{b}^{2} / R^{2}}} .
\end{aligned}
$$

We consider only energies satisfying $8 \pi G_{5} E^{*} z_{b}^{3} \mathrm{e}^{3 z_{b}^{2} / R^{2}}>L^{3}$. For the further analysis of the trapped surface formation, we must obtain the solution of system (43) in an explicit form. A nontrivial solution of the system (we recall that $z_{a}, z_{*}$ and $z_{b}$ are positive) has the form

$$
\begin{aligned}
& z_{a}=\frac{R}{\sqrt{2}} \sqrt{W_{\mathrm{A}}}, \\
& z_{*}=\frac{R}{\sqrt{3}} \sqrt{1+W\left(\frac{-\left(z_{a}^{3} R^{2}-3 z_{a}^{3} z_{b}^{2}-3 z_{b}^{3} z_{a}^{2}+z_{b}^{3} R^{2}\right) \mathrm{e}^{3\left(z_{a}^{2}+z_{b}^{2}\right) / R^{2}}}{R^{2}\left(z_{a}^{3} \mathrm{e}^{3 z_{a}^{2} / R^{2}}+z_{b}^{3} \mathrm{e}^{3 z_{b}^{2} / R^{2}}\right) \mathrm{e}}\right)} \text {, }
\end{aligned}
$$

where

$$
W_{\mathrm{A}}=W\left(2\left(\frac{\left(L^{3} z_{b}^{3} / R^{3}\right) \mathrm{e}^{3 z_{b}^{2} / R^{2}}}{8 \pi G_{5} E^{*} z_{b}^{3} \mathrm{e}^{3 z_{b}^{2} / R^{2}}-L^{3}}\right)^{2 / 3}\right)
$$

and $W(z)$ is Lambert $W$-function.

The dependencies of $z_{a}$ and $z_{*}$ on $z_{b}$ defined by formulas (44), (45), are shown in Figs. 9A. and 9B. at the respective energy values $E=220 \mathrm{GeV}$ and $E=2 \mathrm{GeV}$. We see that $z_{a}$ tends to its the lowest value at infinitely large $z_{b}$. In this limit $z_{a}$ and $z_{*}$ are given by

$$
\left.z_{a}\right|_{z_{b} \rightarrow \infty}=\frac{R}{\sqrt{2}} \sqrt{W_{\mathrm{AM}}},\left.\quad z_{*}\right|_{z_{b} \rightarrow \infty}=\frac{R}{\sqrt{3}} \sqrt{1+W\left(\frac{\left(3 z_{a}^{2}-R^{2}\right) \mathrm{e}^{3 z_{a}^{2} / R^{2}}}{\mathrm{e} R^{2}}\right)},
$$




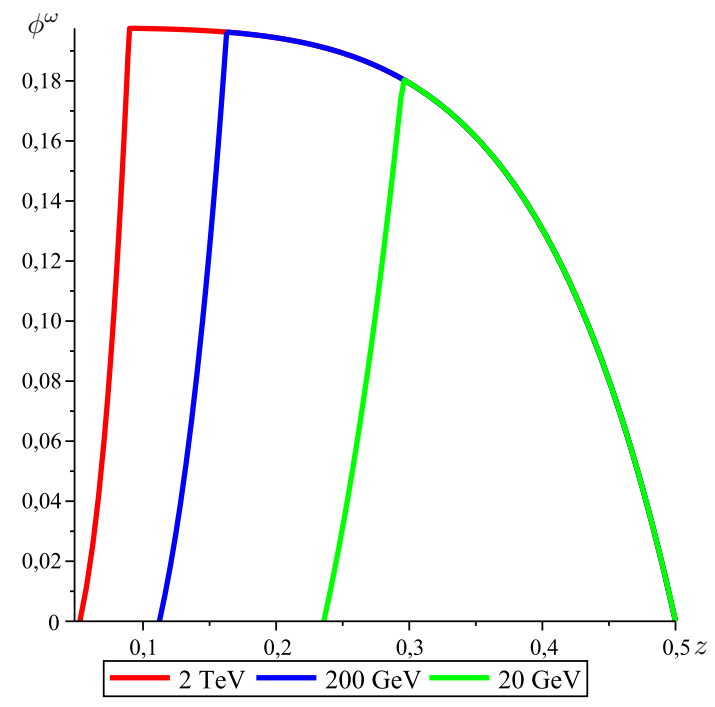

Figure 10: The profiles $\phi^{\omega}$ corresponding to $b(z)=\left(\frac{L}{z}\right) \exp \left(-\frac{z^{2}}{R^{2}}\right)$ at $z_{b}=0.5 \mathrm{fm}, R=1$ fm, $E=20 \mathrm{GeV}, E=200 \mathrm{GeV}, E=2000 \mathrm{GeV}$.

where

$$
W_{\mathrm{AM}}=W\left(\frac{L^{2}}{2\left(\pi G_{5} E^{*}\right)^{2 / 3} R^{2}}\right) .
$$

In this case, the profile is presented in Fig. (10).

Due to the condition $z_{a}<z_{b}$ we found the range of the energies at which black hole is created

$$
E>\frac{L^{5}}{4 \pi G_{5} z_{b}^{3}} \exp \left(-\frac{3 z_{b}^{2}}{R^{2}}\right) .
$$

Black hole is not created already at the energy

$$
E=\frac{L^{5}}{4 \pi G_{5} z b^{3}} \exp \left(-\frac{3 z_{b}^{2}}{R^{2}}\right) .
$$

For the parameters $G_{5}=L^{3} / 1.9, L=4.4 \mathrm{fm}$ and $z_{b}=0.5 \mathrm{fm}$ it equals to $2.2123 \mathrm{GeV}$ (see Fig. (11).

In general, the relative area of the trapped surface depends on the energy and on $z_{b}$ as

$$
s=\frac{L^{3}}{2 G_{5}}\left(-\frac{1}{2 \exp \left(\frac{3 z_{b}^{2}}{R^{2}}\right) z_{b}^{2}}+\frac{1}{2 \exp \left(\frac{3 z_{a}^{2}}{R^{2}}\right) z_{a}^{2}}+\frac{3 \operatorname{Ei}\left(1, \frac{3 z_{b}^{2}}{R^{2}}\right)}{2 R^{2}}-\frac{3 \operatorname{Ei}\left(1, \frac{3 z_{a}^{2}}{R^{2}}\right)}{2 R^{2}}\right),
$$

where $z_{a}$ depends on $z_{b}$ with formulas (44), (45) and $\operatorname{Ei}(1, x)$ is the exponential integral. The dependence of the relative area of the trapped surface is shown graphically at the energies $E=220 \mathrm{GeV}$ and $E=2 \mathrm{GeV}$ in Fig. 12, It can be seen that the maximum value of the trapped surface area $s$ is attained at infinite $z_{b}$ :

$$
\left.s\right|_{z_{b} \rightarrow \infty}=\frac{3}{4} \frac{L^{3}}{G_{5} R^{2}}\left(-\operatorname{Ei}\left(1, \frac{3 z_{a}^{2}}{R^{2}}\right)+\frac{1}{3} \frac{R^{2}}{\exp \left(\frac{3 z_{a}^{2}}{R^{2}}\right) z_{a}^{2}}\right),
$$




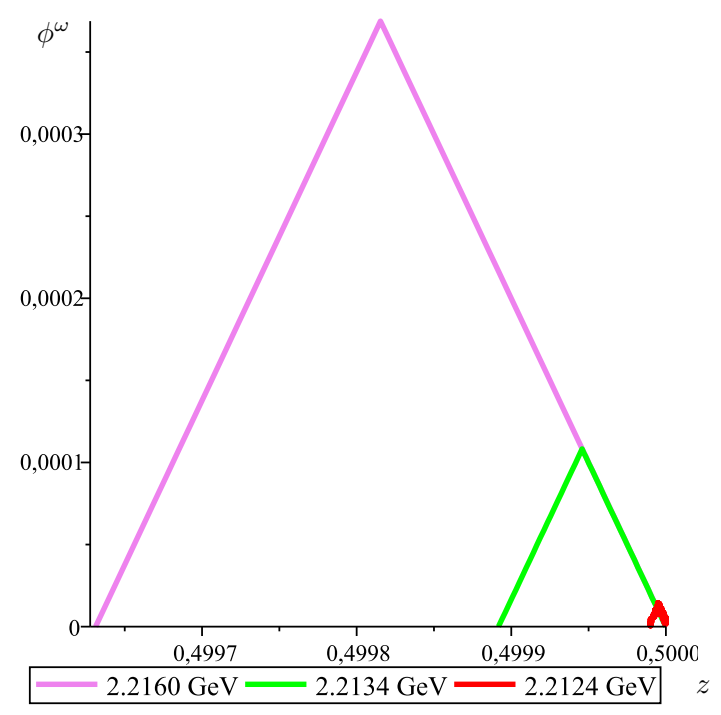

Figure 11: The profiles $\phi^{\omega}$ corresponding to $b(z)=\left(\frac{L}{z}\right) \exp \left(-\frac{z^{2}}{R^{2}}\right)$ at $z_{b}=0.5 \mathrm{fm}, R=1$ fm, $E=2.2160 \mathrm{GeV}, E=2.2134 \mathrm{GeV}, E=2.2160 \mathrm{GeV}$.
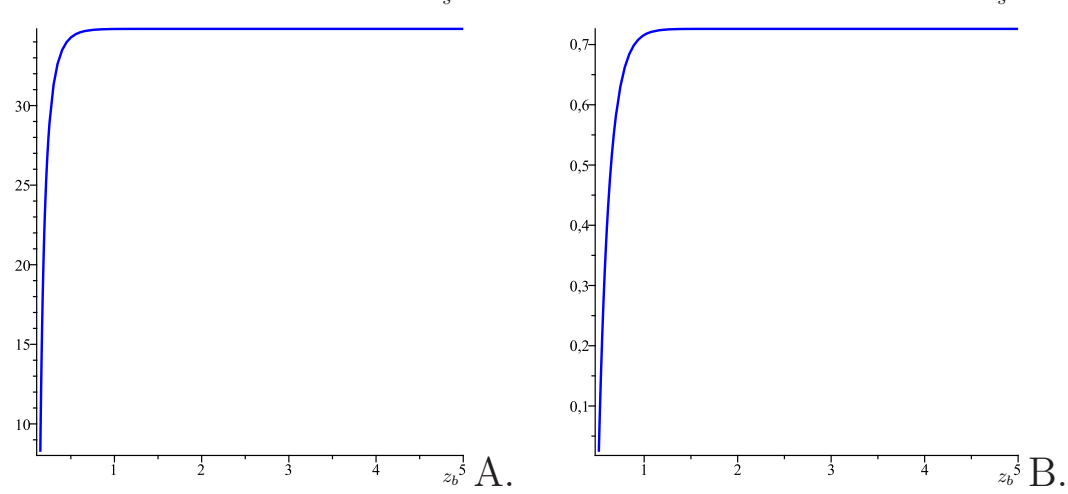

Figure 12: The dependence $s$ on $z_{b}$ at $E=220 \mathrm{GeV}$ (A.) and $E=2 \mathrm{GeV}$ (B.).

where $z_{a}$ is given by (46). We note that the expression in the parentheses is always positive.

Fig. 13 shows the dependence of the relative area of the trapped surface on the energy (at law energies in Fig. 13 A. and hight energies in Fig. 13B.). Fig. 13B. also shows the function $E^{2 / 3}(1+0.007 \ln E)-3$ approximating the obtained dependence at $10 \mathrm{GeV} \lesssim E<1 \mathrm{TeV}$.

\section{Mixed factor of the form $b=\left(\frac{L}{z}\right)^{a} \exp \left(-\frac{z^{2}}{R^{2}}\right)$}

The equation of the domain-wall wave profile in the space with the mixed $b$-factor of the form $b=\left(\frac{L}{z}\right)^{a} \exp \left(-\frac{z^{2}}{R^{2}}\right)$ is written as

$$
\left(\partial_{z}^{2}+\frac{3 b^{\prime}}{b} \partial_{z}\right) \phi^{w}(z)=-\frac{16 \pi G_{5} E}{L^{2}} \frac{\delta\left(z-z_{*}\right)}{b^{3}(z)} .
$$



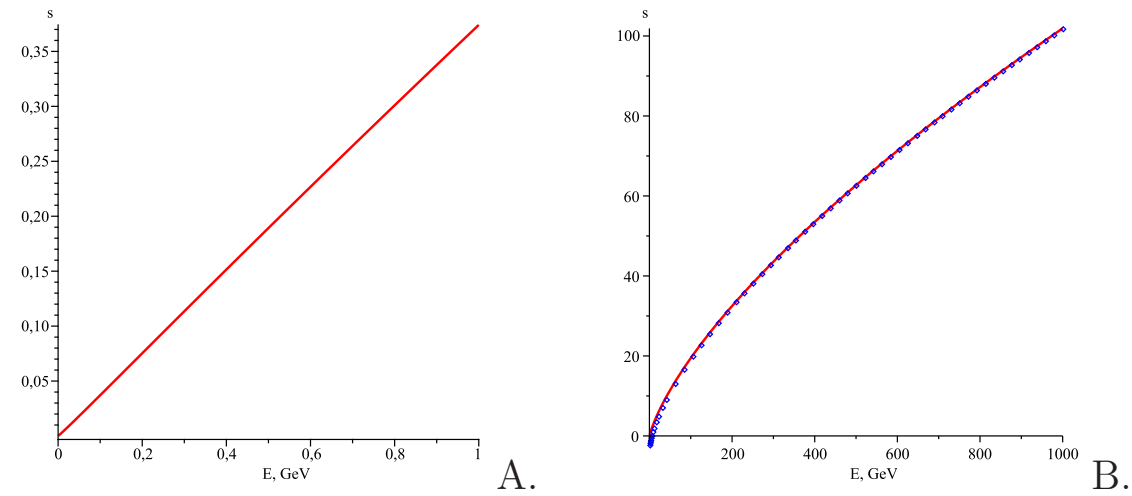

Figure 13: The dependence of the relative area of the trapped surface on energy at low energies (Fig. A. at $0<E<1 \mathrm{GeV}$ ) and at high energies (Fig. B. at $0<E<1000$ $\mathrm{GeV})$ for $b=(L / z) \mathrm{e}^{-z^{2} / R^{2}}$. The function approximating the calculated dependence $E^{2 / 3}(1+$ $0.007 \ln E)-3$ is shown in bold dashes.

As in the cases considered above, the solution of (48) is given as

$$
\phi^{w}(z)=\phi_{a} \Theta\left(z_{*}-z\right)+\phi_{b} \Theta\left(z-z_{*}\right),
$$

where

$$
\begin{aligned}
& \phi_{a}=C_{a} \int_{z_{a}}^{z} b^{-3} d z \\
& \phi_{b}=C_{b} \int_{z_{b}}^{z} b^{-3} d z .
\end{aligned}
$$

The constants $C_{a}$ and $C_{b}$ can be represented in the form

$$
\begin{aligned}
& C_{a}=\frac{16 \pi G_{5} E}{L^{2}} \frac{\int_{z_{b}}^{z_{*}} b^{-3} d z}{\int_{z_{b}}^{z_{a}} b^{-3} d z}, \\
& C_{b}=\frac{16 \pi G_{5} E}{L^{2}} \frac{\int_{z_{a}}^{z_{*}} b^{-3} d z}{\int_{z_{b}}^{z_{a}} b^{-3} d z} .
\end{aligned}
$$

Using the conditions of the trapped surface formation, we get

$$
\begin{aligned}
& \frac{8 \pi G_{5} E}{L^{2}} b^{-3}\left(z_{a}\right) \frac{\int_{z_{b}}^{z_{*}} b^{-3} d z}{\int_{z_{b}}^{z_{a}} b^{-3} d z}=1, \\
& \frac{8 \pi G_{5} E}{L^{2}} b^{-3}\left(z_{b}\right) \frac{\int_{z_{a}}^{z_{*}} b^{-3} d z}{\int_{z_{b}}^{z_{a}} b^{-3} d z}=-1 .
\end{aligned}
$$

Using the designation $\int_{z_{i}}^{z_{j}} b^{-3} d z=F\left(z_{j}\right)-F\left(z_{i}\right)$ and equations (154), (155), we obtain the relations between points $z_{*}, z_{a}, z_{b}$ and $z_{a}, z_{b}$ accordingly

$$
F\left(z_{*}\right)=\frac{b^{-3}\left(z_{b}\right) F\left(z_{a}\right)+b^{-3}\left(z_{a}\right) F\left(z_{b}\right)}{b^{-3}\left(z_{a}\right)+b^{-3}\left(z_{b}\right)},
$$




$$
b^{-3}\left(z_{a}\right)=\frac{b^{-3}\left(z_{b}\right)}{\frac{8 \pi G_{5} E}{L^{2}} b^{-3}\left(z_{b}\right)-1} .
$$

Relation (57) for the factor $b(z)=\left(\frac{L}{z}\right)^{a} \exp \left(-\frac{z^{2}}{R^{2}}\right)$ can be represented in the form

$$
\left(\frac{z_{a}}{L}\right)^{3 a} \exp \left(\frac{3 z_{a}^{2}}{R^{2}}\right)=\frac{\left(\frac{z_{b}}{L}\right)^{3 a} \exp \left(\frac{3 z_{b}^{2}}{R^{2}}\right)}{\frac{8 \pi G_{5} E}{L^{2}}\left(\frac{z_{b}}{L}\right)^{3 a} \exp \left(\frac{3 z_{b}^{2}}{R^{2}}\right)-1},
$$

with a solution

$$
z_{a}=\sqrt{\frac{a R^{2}}{2} \mathrm{~W}\left(\frac{2 L^{2}}{a R^{2}} \exp \left(\frac{2 z_{b}^{2}}{a R^{2}}\right)\left(\frac{\left(\frac{z_{b}}{L}\right)^{3 a} L^{2}}{8 \pi G_{5} E\left(\frac{z_{b}}{L}\right)^{3 a} \exp \left(\frac{3 z_{b}^{2}}{R^{2}}\right)-L^{2}}\right)^{\frac{2}{3 a}}\right)},
$$

one may rewrite (59) in the equivalent form as

$$
z_{a}=R \sqrt{\frac{a}{2}} \sqrt{\mathrm{W}\left(\frac{2 L^{2}}{a R^{2}}\left(\frac{\left(\frac{z_{b}}{L}\right)^{3 a} \exp \left(\frac{3 z_{b}^{2}}{R^{2}}\right)}{\frac{8 \pi G_{5} E}{L^{2}}\left(\frac{z_{b}}{L}\right)^{3 a} \exp \left(\frac{3 z_{b}^{2}}{R^{2}}\right)-1}\right)^{\frac{2}{3 a}}\right)}
$$

This relation has the simplest form at $a=1 / 3$, namely

$$
z_{a}=R \sqrt{\frac{1}{6}} \sqrt{\mathrm{W}\left(\frac{6 L^{2}}{R^{2}}\left(\frac{\left(\frac{z_{b}}{L}\right) \exp \left(\frac{3 z_{b}^{2}}{R^{2}}\right)}{\frac{8 \pi G_{5} E}{L^{2}}\left(\frac{z_{b}}{L}\right) \exp \left(\frac{3 z_{b}^{2}}{R^{2}}\right)-1}\right)^{2}\right)} .
$$

The behavior of $z_{a}$ is presented at fixed energies in Fig. 16. When $z_{b} \rightarrow \infty$, we obtain

$$
\begin{gathered}
\left(\frac{z_{a}}{L}\right)^{3 a} \exp \left(\frac{3 z_{a}^{2}}{R^{2}}\right) \rightarrow \frac{L^{2}}{8 \pi G_{5} E}, \\
\left.z_{a}\right|_{z_{b} \rightarrow \infty}=R \sqrt{\frac{a}{2}} \sqrt{\mathrm{W}\left(\frac{2 L^{2}}{a R^{2}}\left(\frac{L^{2}}{8 \pi G_{5} E}\right)^{\frac{2}{3 a}}\right) .}
\end{gathered}
$$

Substituting (57) to (56), we obtain the relation

$$
\frac{8 \pi G_{5} E}{L^{2}} b^{-3}\left(z_{b}\right)=\frac{\Gamma\left(\frac{3 a+1}{2},-\frac{3 z_{b}^{2}}{R^{2}}\right)-\Gamma\left(\frac{3 a+1}{2},-\frac{3 z_{a}^{2}}{R^{2}}\right)}{\Gamma\left(\frac{3 a+1}{2},-\frac{3 z_{*}^{2}}{R^{2}}\right)-\Gamma\left(\frac{3 a+1}{2},-\frac{3 z_{a}^{2}}{R^{2}}\right)} .
$$

For the case of $a=1 / 3$ it is written as

$$
\frac{8 \pi G_{5} E}{L^{2}} b^{-3}\left(z_{b}\right)=\frac{\exp \left(\frac{3 z b^{2}}{R^{2}}\right)-\exp \left(\frac{3 z a^{2}}{R^{2}}\right)}{\exp \left(\frac{3 z_{*}^{2}}{R^{2}}\right)-\exp \left(\frac{3 z a^{2}}{R^{2}}\right)} .
$$


Applying to (64) the $\Gamma$ function property

$$
\Gamma(A, X)=\Gamma(A)-\frac{X^{A}{ }_{1} F_{1}(A, A+1,-X)}{A},
$$

we have

$$
\frac{8 \pi G_{5} E}{L^{2}} b^{-3}\left(z_{b}\right)=\frac{z_{a}^{\frac{3 a+1}{2}}{ }_{1} F_{1}\left(\frac{3 a+1}{2}, \frac{3 a+3}{2}, \frac{3 z_{a}^{2}}{R^{2}}\right)-z_{b}^{\frac{3 a+1}{2}}{ }_{1} F_{1}\left(\frac{3 a+1}{2}, \frac{3 a+3}{2}, \frac{3 z_{b}^{2}}{R^{2}}\right)}{z_{a}^{\frac{3 a+1}{2}}{ }_{1} F_{1}\left(\frac{3 a+1}{2}, \frac{3 a+3}{2}, \frac{3 z_{a}^{2}}{R^{2}}\right)-z_{*}^{\frac{3 a+1}{2}}{ }_{1} F_{1}\left(\frac{3 a+1}{2}, \frac{3 a+3}{2}, \frac{3 z_{*}^{2}}{R^{2}}\right)} .
$$

With the help of the relation

$$
{ }_{1} F_{1}(\alpha, \gamma, z)=\sum_{k=0}^{\infty} \frac{(\alpha)_{k}}{(\gamma)_{k}} \frac{z^{k}}{k !}
$$

where ${ }_{1} F_{1}(\alpha, \gamma, z)$ is the confluent hypergeometric function, $(\alpha)_{k}$ and $(\gamma)_{k}$ are the pochhammer symbols, we have

$$
\frac{8 \pi G_{5} E}{L^{2}}\left(\frac{z_{b}}{L}\right)^{3 a} \exp \left(\frac{3 z_{b}^{2}}{R^{2}}\right)=\sum_{k=0}^{\infty} \frac{\left(\frac{3 z_{a}^{2}}{R^{2}}\right)^{k+\frac{3 a+1}{2}}-\left(\frac{3 z_{b}^{2}}{R^{2}}\right)^{k+\frac{3 a+1}{2}}}{\left(\frac{3 z_{a}^{2}}{R^{2}}\right)^{k+\frac{3 a+1}{2}}-\left(\frac{3 z_{*}^{2}}{R^{2}}\right)^{k+\frac{3 a+1}{2}}} .
$$

Accordingly to (69) we observe that $z_{*} \rightarrow z_{a}$ from the right side, if $z_{b} \rightarrow \infty$.

For an arbitrary $a$ the relation (56) can be represented in the form:

$$
\begin{gathered}
z_{* 1}^{2 a} F_{1}\left(\frac{3 a+1}{2} ; \frac{3(a+1)}{2} ; \frac{3 z_{*}^{2}}{R^{2}}\right)= \\
=\frac{z_{a}^{2 a} z_{b}^{2 a}\left(z_{b}^{a} e^{\left(\frac{3 z_{b}^{2}}{R^{2}}\right)}{ }_{1} F_{1}\left(\frac{3 a+1}{2} ; \frac{3(a+1)}{2} ; \frac{3 z_{a}^{2}}{R^{2}}\right)+z_{a}^{a} e^{\left(\frac{3 z_{a}^{2}}{R^{2}}\right)}{ }_{1} F_{1}\left(\frac{3 a+1}{2} ; \frac{3(a+1)}{2} ; \frac{3 z_{b}^{2}}{R^{2}}\right)\right)}{z_{a}^{3 a} \exp \left(\frac{3 z_{a}^{2}}{R^{2}}\right)+z_{b}^{3 a} \exp \left(\frac{3 z_{B}^{2}}{R^{2}}\right)} .
\end{gathered}
$$

This equation is rather difficult for analytic solutions. However, in the case $a=1 / 3$ the expression (56) can be represented as the following one

$$
\exp \left(\frac{3 z_{*}^{2}}{R^{2}}\right)=\frac{z_{a}+z_{b}}{z_{a} \exp \left(-\frac{3 z_{b}^{2}}{R^{2}}\right)+z_{b} \exp \left(-\frac{3 z_{a}^{2}}{R^{2}}\right)}
$$

which has the analytical solution

$$
z_{*}=\sqrt{\frac{R^{2}}{3} \ln \left(\frac{z_{a}+z_{b}}{z_{a} \exp \left(-\frac{3 z_{b}^{2}}{R^{2}}\right)+z_{b} \exp \left(-\frac{3 z_{a}^{2}}{R^{2}}\right)}\right)} .
$$


The functions $\phi_{a}, \phi_{b}$ can be represented such as

$$
\begin{aligned}
\phi_{a} & =\frac{16 \pi G_{5} E}{L^{2}} \cdot \frac{\int_{z_{b}}^{z_{*}} b^{-3} d z \cdot \int_{z_{a}}^{z} b^{-3} d z}{\int_{z_{b}}^{z_{a}} b^{-3} d z} \\
\phi_{b} & =\frac{16 \pi G_{5} E}{L^{2}} \cdot \frac{\int_{z_{a}}^{z_{*}} b^{-3} d z \cdot \int_{z_{b}}^{z} b^{-3} d z}{\int_{z_{b}}^{z_{a}} b^{-3} d z} .
\end{aligned}
$$

Considering the integral

$$
\int b^{-3} d z=\left(\frac{3^{-\frac{1}{2}-\frac{3 a}{2}}}{2}\right)\left(-\frac{z^{2}}{R^{2}}\right)^{-\frac{1}{2}-\frac{3 a}{2}}\left(\frac{L}{z}\right)^{-3 a} z\left(\Gamma\left(\frac{3 a}{2}+\frac{1}{2}\right)-\Gamma\left(\frac{3 a}{2}+\frac{1}{2},-\frac{3 z^{2}}{R^{2}}\right)\right)+C,
$$

and using the property (66), one obtains

$$
\int b^{-3} d z=\frac{z\left(\frac{L}{z}\right)^{-3 a}{ }_{1} F_{1}\left(\frac{3 a+1}{2}, \frac{3(a+1)}{2}, \frac{3 z^{2}}{R^{2}}\right)}{3 a+1}+C .
$$

Introducing new designation

$$
\Upsilon(z)=z\left(\frac{L}{z}\right)^{-3 a}{ }_{1} F_{1}\left(\frac{3 a+1}{2}, \frac{3(a+1)}{2}, \frac{3 z^{2}}{R^{2}}\right),
$$

So the expressions (171) and (72) are represented in the form

$$
\begin{aligned}
\phi_{a}=\frac{16 \pi G_{5} E}{(3 a+1) L^{2}} \frac{\left(\Upsilon\left(z_{b}\right)-\Upsilon\left(z_{*}\right)\right)\left(\Upsilon(z)-\Upsilon\left(z_{a}\right)\right)}{\Upsilon\left(z_{b}\right)-\Upsilon\left(z_{a}\right)}, \\
\phi_{b}=\frac{16 \pi G_{5} E}{(3 a+1) L^{2}} \frac{\left(\Upsilon\left(z_{*}\right)-\Upsilon\left(z_{a}\right)\right)\left(\Upsilon\left(z_{b}\right)-\Upsilon(z)\right)}{\Upsilon\left(z_{b}\right)-\Upsilon\left(z_{a}\right)} .
\end{aligned}
$$

The expression (74) has the following form at $a=1 / 3$

$$
\Upsilon(z)=\frac{R^{2}}{3 L}\left(\exp \left(\frac{3 z^{2}}{R^{2}}\right)-1\right)
$$

so the functions $\phi_{a}$ and $\phi_{b}$ are represented as

$$
\begin{aligned}
& \phi_{a}=\frac{8}{3} \frac{\pi G_{5} E R^{2}}{L^{3}} \frac{\left(\exp \left(\frac{3 z_{b}^{2}}{R^{2}}\right)-\exp \left(\frac{3 z_{*}^{2}}{R^{2}}\right)\right)\left(\exp \left(\frac{3 z^{2}}{R^{2}}\right)-\exp \left(\frac{3 z_{a}^{2}}{R^{2}}\right)\right)}{\exp \left(\frac{3 z_{b}^{2}}{R^{2}}\right)-\exp \left(\frac{3 z_{a}^{2}}{R^{2}}\right)}, \\
& \phi_{b}=\frac{8}{3} \frac{\pi G_{5} E R^{2}}{L^{3}} \frac{\left(\exp \left(\frac{3 z_{*}^{2}}{R^{2}}\right)-\exp \left(\frac{3 z_{a}^{2}}{R^{2}}\right)\right)\left(\exp \left(\frac{3 z_{b}^{2}}{R^{2}}\right)-\exp \left(\frac{3 z^{2}}{R^{2}}\right)\right)}{\exp \left(\frac{3 z_{b}^{2}}{R^{2}}\right)-\exp \left(\frac{3 z_{a}^{2}}{R^{2}}\right)} .
\end{aligned}
$$




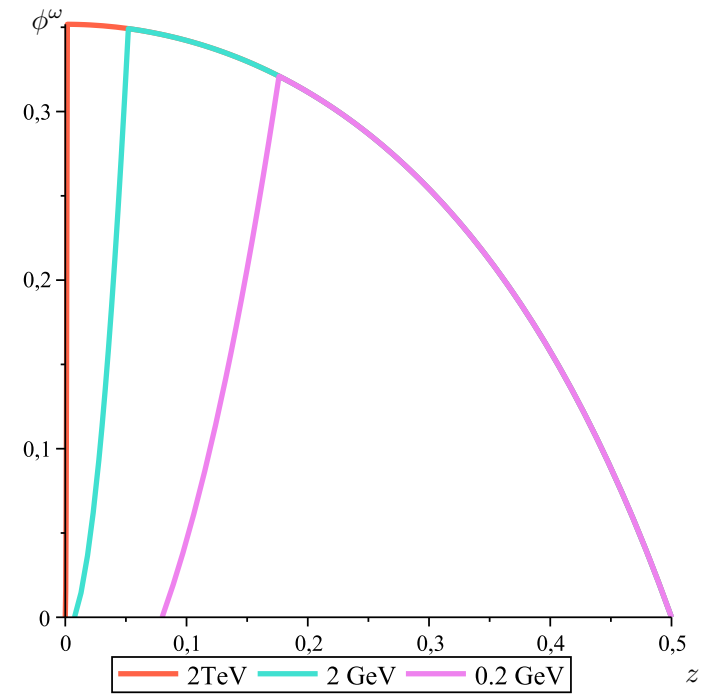

Figure 14: The profile $\phi^{\omega}$ corresponding to $b(z)=\left(\frac{L}{z}\right)^{a} \exp \left(-\frac{z^{2}}{R^{2}}\right)$ at $z_{b}=0.5 \mathrm{fm}, a=1 / 3$, $R=1 \mathrm{fm}, E=0.2 \mathrm{GeV}, E=2 \mathrm{GeV}, E=2 \mathrm{TeV}$.

The given profiles at $a=1 / 3, G_{5}=L^{3} / 1.9, L=4.4 \mathrm{fm}, z_{b}=0.5 \mathrm{fm}$ are presented in Fig. (14).

Similar to the case discussed in the sections above, due to the condition $z_{a}<z_{b}$ we find the range of the energies at which black hole is created

$$
E>\frac{L^{3}}{4 \pi G_{5} z_{b} \exp \left(\frac{3 z_{b}^{2}}{R^{2}}\right)} .
$$

Black hole is not created created as the energy approaches to the value

$$
E=\frac{L^{3}}{4 \pi G_{5} z_{b} \exp \left(\frac{3 z_{b}^{2}}{R^{2}}\right)},
$$

which is equal to $28.568 \mathrm{MeV}$ for the parameters $G_{5}=L^{3} / 1.9, L=4.4 \mathrm{fm}, a=1 / 3$ and $z_{b}=0.5$ fm., (see Fig. [15).

In considering case the relative trapped surface area is

$$
s=\left.\frac{\left(\frac{L}{z}\right)^{3 a} z \exp \left(-\frac{3 z^{2}}{2 R^{2}}\right)\left(2\left(\frac{3 z^{2}}{R^{2}}\right)^{\frac{3 a-1}{4}} \mathbf{M}\left(\frac{-3 a+1}{4}, \frac{3(-a+1)}{4}, \frac{3 z^{2}}{R^{2}}\right)+3(1-a) \exp \left(-\frac{3 z^{2}}{2 R^{2}}\right)\right)}{2 G_{5} \cdot 3(3 a-1)(a-1)}\right|_{z_{a}} ^{z_{b}},
$$

where $\mathbf{M}(\mu, \nu, z)=\exp \left(-\frac{z}{2}\right) z^{\frac{1}{2}+\nu}{ }_{1} F_{1}\left(\frac{1}{2}+\nu-\mu, 1+2 \nu, z\right)$ is the Whittaker function, $a \neq 1 / 3$, $a \neq 1$.

We graphically represent $s$ as a function of $z_{b}$ in Fig. 17 at energies $2 \mathrm{GeV}$ and 220 $\mathrm{GeV}$. Hence, it is clear that the relative trapped surface area tends to its maximum value at infinite $z_{b}$ :

$$
s \rightarrow \frac{\left(\frac{L}{z_{a}}\right)^{3 a} z_{a} \exp \left(-\frac{3 z_{a}^{2}}{2 R^{2}}\right)\left(2\left(\frac{3 z_{a}^{2}}{R^{2}}\right)^{\frac{3 a-1}{4}} \mathbf{M}\left(\frac{-3 a+1}{4}, \frac{3(-a+1)}{4}, \frac{3 z_{a}^{2}}{R^{2}}\right)+3(1-a) \exp \left(-\frac{3 z_{a}^{2}}{2 R^{2}}\right)\right)}{6 G_{5} \cdot(3 a-1)(1-a)}
$$




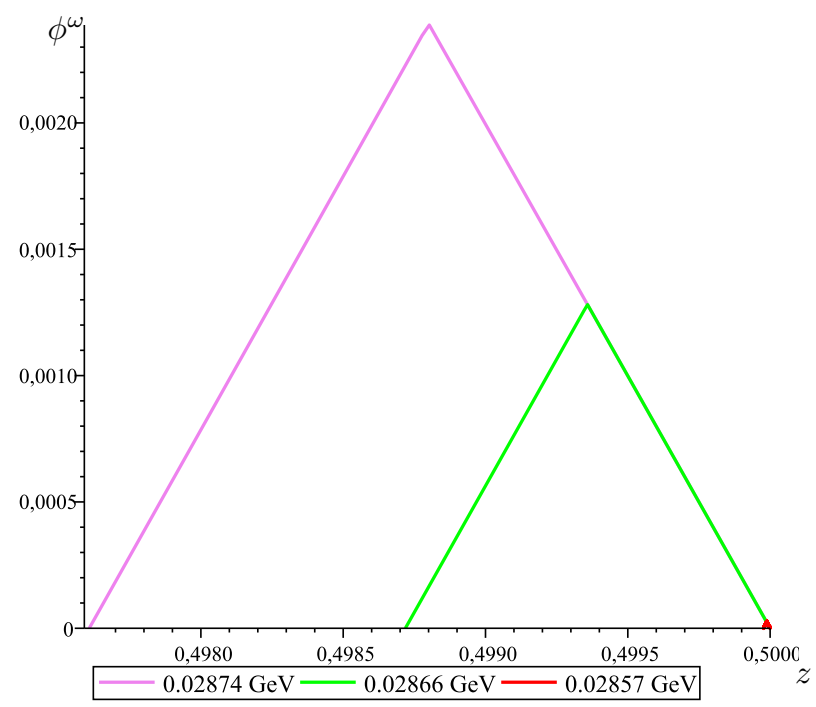

Figure 15: The profiles $\phi^{\omega}$ corresponding to $b(z)=\left(\frac{L}{z}\right)^{a} \exp \left(-\frac{z^{2}}{R^{2}}\right)$ at $z_{b}=0.5 \mathrm{fm}, a=1 / 3$, $R=1 \mathrm{fm}, E=28.74 \mathrm{MeV}, E=28.66 \mathrm{MeV}, E=28.57 \mathrm{MeV}$.

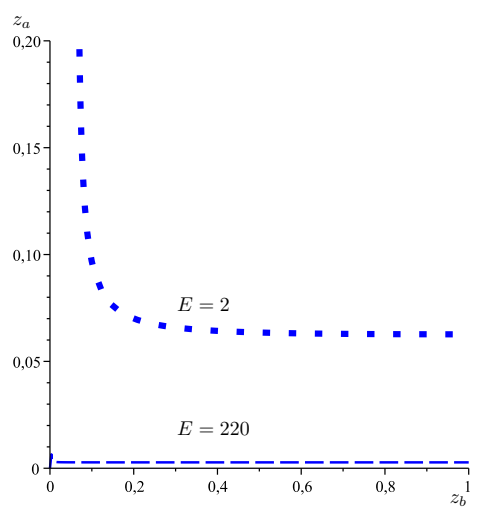

Figure 16: The dependence of $z_{a}$ on $z_{b}$ corresponding to $a=1 / 2$ at energies $2 \mathrm{GeV}$ and 220 $\mathrm{GeV}$.

where $a>\frac{1}{3}, a \neq 1, z_{a}$ is defined by (63).

For the case corresponding to $a=1 / 3$ the relative trapped surface area is written as

$$
s=-\left.\frac{L}{4 G_{5}} \operatorname{Ei}\left(1, \frac{3 z^{2}}{R^{2}}\right)\right|_{z_{a}} ^{z_{b}},
$$

and

$$
s \rightarrow \frac{L}{4 G_{5}} \operatorname{Ei}\left(1, \frac{3 z_{a}^{2}}{R^{2}}\right)
$$

at $z_{b} \rightarrow \infty$. 


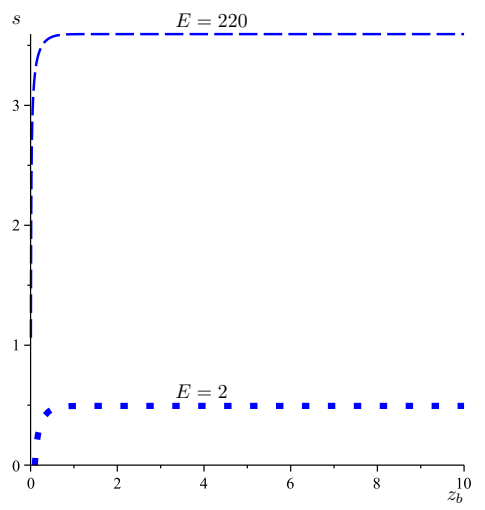

Figure 17: The dependence of the relative area of the trapped surface on $z_{b}$ corresponding to $a=1 / 2$ at energies $2 \mathrm{GeV}$ and $220 \mathrm{GeV}$.

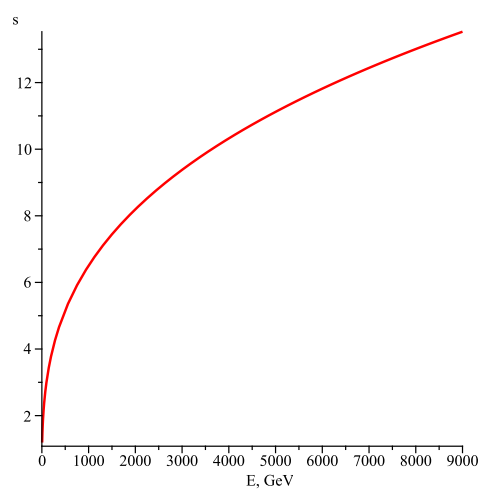

Figure 18: The dependence of the relative area of the trapped surface on energy corresponding to $a=1 / 2$.

\section{Conclusion}

We have investigated the possibility of the black hole formation in the domain-wall collisions in modified $\mathrm{AdS}_{5}$ spaces with $b$-factors. We considered several types of $b$-factor: power-law, exponential and mixed. We analyzed the dependence of entropy on the energy of colliding ions in the spaces with $b$-factors based on the analysis of the conditions for forming the trapped surfaces. With the AdS/CFT duality taken into account, the obtained results allow modeling the dependence of multiplicity of the produced particles on the energy of the colliding heavy-ions. We note that the results for the power-law factors are agree with the conclusion in the previously examined cases of central collisions of pointlike sources. The exponential factors of the collision domains do not lead to additional logarithms which take place in the case of central collisions of point sources [14] in the presence of the exponential $b$-factors; nevertheless, additional logarithms appear when the mixed factors are considered. The derived results can be used to compare with the experimental curves for the multiplicity of particle formation in heavy-ion collisions. 

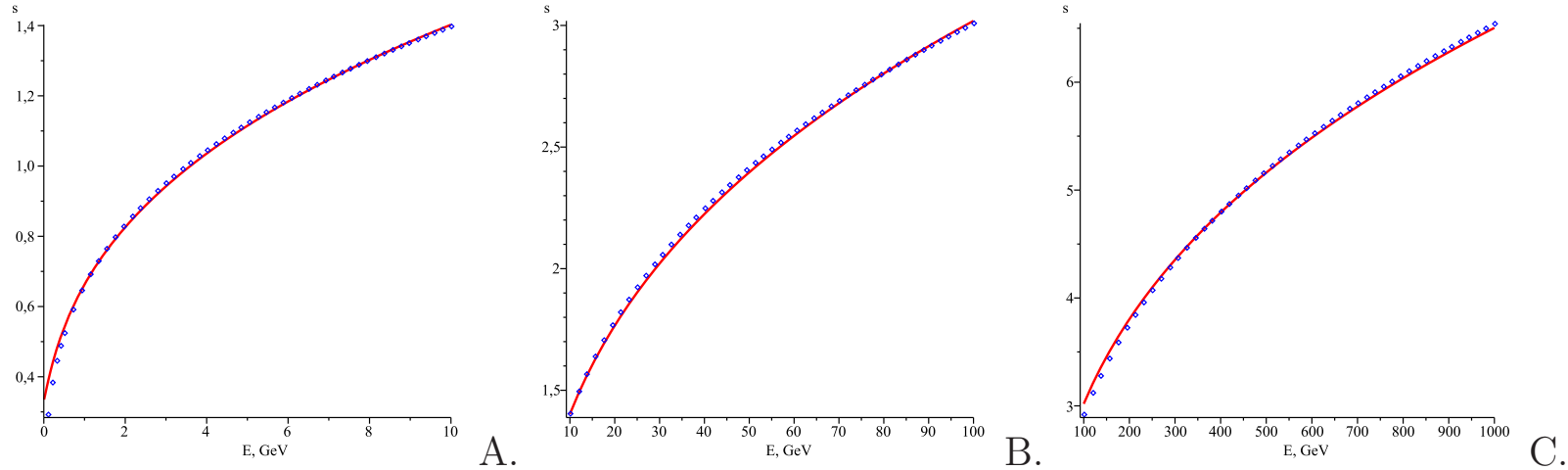

Figure 19: The dependence of the relative area of the trapped surface on energy is shown in red line and the function approximating the calculated dependence corresponding to $a=1 / 2$ is shown in blue points (Fig. A. $\frac{E^{0.3}(57-29.75(\ln (E+100)))-7}{2 G_{5}}$ at $0<E<10 \mathrm{CeV}$ ), (Fig. B. $\frac{E^{0.3}(61-45.05(\ln (E+100)))-24}{2 G_{5}}$ at $\left.10<E<100 \mathrm{GeV}\right)$ and (Fig. C. $\frac{E^{0.3}(81-5.95 \ln (E+100))-67}{2 G_{5}}$ at $\left.10^{2}<E<10^{3} \mathrm{GeV}\right)$.

\section{Acknowledgments}

The results introduced in this paper were partially presented by one of the authors (I. Ya. A.) at the Fourth International Conference "Models in Quantum Field Theory" (MQFT-2012) devoted to the memory of A. N. Vassiliev.

This work was partially supported by the Russian Foundation for Basic Research (grants 11-01-00894-a (I. A., E. P.) and 1202-31109-mol_a (E. P., T. P.)) and by the Russian Ministry of Education and Science under grant NSh-3042.2014.2 (E.P.).

\section{References}

[1] J. M. Maldacena, "The Large N limit of superconformal field theories and supergravity," Adv. Theor. Math. Phys. 2, 231 (1998) [hep-th/9711200].

[2] S. S. Gubser, I. R. Klebanov, A. M. Polyakov, "Gauge theory correlators from noncritical string theory," Phys. Lett. B428, 105-114 (1998) [hep-th/9802109].

[3] E. Witten, "Anti-de Sitter space and holography," Adv. Theor. Math. Phys. 2, 253-291 (1998) [hep-th/9802150].

[4] J. Casalderrey-Solana, H. Liu, D. Mateos, K. Rajagopal and U. A. Wiedemann, "Gauge/String Duality, Hot QCD and Heavy Ion Collisions," arXiv:1101.0618 [hep-th].

[5] I. Aref'eva, "Holography for quark-gluon plasma formation in heavy ion collisions," PoS ICMP 2012, 025 (2012).

[6] S. S. Gubser, S. S. Pufu and A. Yarom, "Entropy production in collisions of gravitational shock waves and of heavy ions," Phys. Rev. D 78, 066014 (2008) arXiv:0805.1551 [hep-th]]. 
S. S. Gubser, S. S. Pufu and A. Yarom, "Off-center collisions in AdS(5) with applications to multiplicity estimates in heavy-ion collisions," JHEP 0911, 050 (2009) arXiv:0902.4062 [hep-th]].

[7] J. L. Albacete, Y. V. Kovchegov and A. Taliotis, "Modeling Heavy Ion Collisions in AdS/CFT," JHEP 0807, 100 (2008) [arXiv:0805.2927 [hep-th]].

[8] L. Alvarez-Gaume, C. Gomez, A. Sabio Vera, A. Tavanfar and M. A. Vazquez-Mozo, "Critical formation of trapped surfaces in the collision of gravitational shock waves," JHEP 0902, 009 (2009) arXiv:0811.3969 [hep-th]].

[9] P. M. Chesler and L. G. Yaffe, "Horizon formation and far-from-equilibrium isotropization in supersymmetric Yang-Mills plasma," Phys. Rev. Lett. 102, 211601 (2009) arXiv:0812.2053 [hep-th]].

[10] S. Lin and E. Shuryak, "Grazing Collisions of Gravitational Shock Waves and Entropy Production in Heavy Ion Collision," Phys. Rev. D 79, 124015 (2009) arXiv:0902.1508 [hep-th]].

S. Lin and E. Shuryak, "On the critical condition in gravitational shock wave collision and heavy ion collisions," Phys. Rev. D 83, 045025 (2011) [arXiv:1011.1918 [hep-th]].

[11] I. Ya. Aref'eva, A. A. Bagrov and E. A. Guseva, "Critical Formation of Trapped Surfaces in the Collision of Non-expanding Gravitational Shock Waves in de Sitter Space-Time," JHEP 0912, 009 (2009) arXiv:0905.1087 [hep-th]].

I. Ya. Aref'eva, A. A. Bagrov and L. V. Joukovskaya, "Critical Trapped Surfaces Formation in the Collision of Ultrarelativistic Charges in (A)dS," JHEP 1003, 002 (2010) arXiv:0909.1294 [hep-th]].

I. Ya. Aref'eva and A. A. Bagrov, "Formation of trapped surfaces in the collision of nonexpanding gravitational shock waves in an $\mathrm{AdS}_{4}$ space-time," Theor. Math. Phys. 161, 1647 (2009).

[12] P. M. Chesler and L. G. Yaffe, "Holography and colliding gravitational shock waves in asymptotically $\mathrm{AdS}_{5}$ spacetime," Phys. Rev. Lett. 106, 021601 (2011) arXiv:1011.3562 [hep-th]].

[13] I. Ya. Aref'eva, A. A. Bagrov and E. O. Pozdeeva, "Holographic phase diagram of quarkgluon plasma formed in heavy-ions collisions," JHEP 1205, 117 (2012) arXiv:1201.6542 [hep-th]].

[14] E. Kiritsis and A. Taliotis, "Multiplicities from black-hole formation in heavy-ion collisions," JHEP 1204, 065 (2012) [arXiv:1111.1931 [hep-ph]].

A. Taliotis, "Extra dimensions, black holes and fireballs at the LHC," JHEP 1305, 034 (2013) arXiv:1212.0528 [hep-th]].

[15] V. Balasubramanian, A. Bernamonti, J. de Boer, N. Copland, B. Craps, E. KeskiVakkuri, B. Muller and A. Schafer et al., "Holographic Thermalization," Phys. Rev. D 84, 026010 (2011) arXiv:1103.2683 [hep-th]].

[16] J. Abajo-Arrastia, J. Aparicio and E. Lopez, "Holographic Evolution of Entanglement Entropy," JHEP 1011, 149 (2010) [arXiv:1006.4090 [hep-th]]. 
[17] R. Callan, J. -Y. He and M. Headrick, "Strong subadditivity and the covariant holographic entanglement entropy formula," JHEP 1206, 081 (2012) arXiv:1204.2309 [hepth]].

[18] I. Ya. Aref'eva and I. V. Volovich, "On Holographic Thermalization and Dethermalization of Quark-Gluon Plasma," arXiv:1211.6041 [hep-th].

[19] K. Aamodt, et al. [ALICE Collaboration], "Charged-particle multiplicity density at mid-rapidity in central Pb-Pb collisions at $\sqrt{s_{N N}}=2.76 \mathrm{TeV}$," Phys. Rev. Lett. 105, 252301 (2010) arXiv:1011.3916 [nucl-ex]].

[20] U. Gursoy and E. Kiritsis, "Exploring improved holographic theories for QCD: Part I," JHEP 0802, 032 (2008) arXiv:0707.1324 [hep-th]].

U. Gursoy, E. Kiritsis and F. Nitti, "Exploring improved holographic theories for QCD: Part II," JHEP 0802, 019 (2008) [arXiv:0707.1349 [hep-th]].

[21] U. Gursoy, E. Kiritsis, L. Mazzanti and F. Nitti, "Deconfinement and Gluon Plasma Dynamics in Improved Holographic QCD," Phys. Rev. Lett. 101, 181601 (2008) arXiv:0804.0899 [hep-th]].

[22] U. Gursoy, E. Kiritsis, L. Mazzanti and F. Nitti, "Holography and Thermodynamics of 5D Dilaton-gravity," JHEP 0905, 033 (2009) [arXiv:0812.0792 [hep-th]].

[23] I. Ya. Aref'eva, A. S. Koshelev and S. Y. .Vernov, "Exactly solvable SFT inspired phantom model," Theor. Math. Phys. 148, 895 (2006) astro-ph/0412619].

[24] I. Ya. Aref'eva, "Catalysis of black holes/wormholes formation in high energy collisions," Phys. Part. Nucl. 41, 835 (2010) arXiv:0912.5481 [hep-th]].

[25] M. Hotta and M. Tanaka, "Shock wave geometry with nonvanishing cosmological constant," Class. Quant. Grav. 10, 307 (1993).

[26] J. Podolsky and J. B. Griffiths, "Nonexpanding impulsive gravitational waves with an arbitrary cosmological constant," Phys. Lett. A 261, 1 (1999) [gr-qc/9908008].

J. Podolsky, "Exact impulsive gravitational waves in space-times of constant curvature", Gravitation: Following the Prague Inspiration, eds. O. Semerák, J. Podolský, and M. Žofka, World Sci., Singapore, 205-246 (2002) [gr-qc/0201029].

[27] K. Sfetsos, "On gravitational shock waves in curved space-times," Nucl. Phys. B 436, 721 (1995) hep-th/9408169].

[28] I. Ya. Aref'eva, A. A. Bagrov, and L. V. Joukovskaya, "Several aspects of applying distributions to analysis of gravitational shock waves in general relativity", St. Petersburg Math. J., 22, 337 (2011).

[29] T. Dray and G. 't Hooft, "The Gravitational Shock Wave of a Massless Particle," Nucl. Phys. B 253, 173 (1985).

V. Ferrari, P. Pendenza and G. Veneziano, "Beamlike Gravitational Waves and Their Geodesics," Gen. Rel. Grav. 20, 1185 (1988). 